Article

\title{
Sustainable Functionalization of PAN to Improve Tinctorial Capacity
}

\author{
Vasilica Popescu ${ }^{1, *}$, Ingrid Ioana Buciscanu ${ }^{1}$, Melinda Pruneanu ${ }^{1}$, Stelian Sergiu Maier ${ }^{1}$, Angela Danila ${ }^{1}$, \\ Vasilica Maier ${ }^{1}$, Marius Pîslaru ${ }^{2}$, Vlad Rotaru ${ }^{1}$, Irina Niculina Cristian ${ }^{3}{ }^{\circledR}$, Andrei Popescu ${ }^{4}$, Bogdan Istrate ${ }^{4}$, \\ Alexandra Cristina Blaga ${ }^{5}$, Florin Ciolacu ${ }^{6}$, Igor Cretescu ${ }^{7}$, Petronela Chelariu ${ }^{1}$ and Marina Marin ${ }^{1}$
}

1 Department of Chemical Engineering in Textiles and Leather, Faculty of Industrial Design and Business Management, “Gheorghe Asachi” Technical University of Iasi, 700050 Iasi, Romania; ingrid-ioana.buciscanu@academic.tuiasi.ro (I.I.B.); melinda.pruneanu@academic.tuiasi.ro (M.P.); stelian-sergiu.maier@academic.tuiasi.ro (S.S.M.); angela.danila@academic.tuiasi.ro (A.D.); vasilica.maier@academic.tuiasi.ro (V.M.); rotaruvlad1980@gmail.com (V.R.); petronelachelariu@yahoo.com (P.C.); marinamarin9817@gmail.com (M.M.)

2 Department of Engineering and Management, Faculty of Industrial Design and Business Management, "Gheorghe Asachi" Technical University of Iasi, 700050 Iasi, Romania; marius.pislaru@academic.tuiasi.ro

3 Design and Engineering of Textile Products, Faculty of Industrial Design and Business Management, "Gheorghe Asachi" Technical University of Iasi, 700050 Iasi, Romania; irina-niculina.cristian@academic.tuiasi.ro

4 Department of Machine Design, Mechatronics and Robotics, Faculty of Mechanical Engineering, "Gheorghe Asachi" Technical University of Iasi, 700050 Iasi, Romania; andrei.popescu@academic.tuiasi.ro (A.P.); bogdan.istrate@academic.tuiasi.ro (B.I.)

check for updates

Citation: Popescu, V.; Buciscanu, I.I.; Pruneanu, M.; Maier, S.S.; Danila, A.; Maier, V.; Pîslaru, M.; Rotaru, V.;

Cristian, I.N.; Popescu, A.; et al. Sustainable Functionalization of PAN to Improve Tinctorial Capacity. Polymers 2021, 13, 3665. https:// doi.org/10.3390/polym13213665

Academic Editor: Vijay

Kumar Thakur

Received: 22 September 2021

Accepted: 16 October 2021

Published: 24 October 2021

Publisher's Note: MDPI stays neutral with regard to jurisdictional claims in published maps and institutional affiliations.

Copyright: (C) 2021 by the authors. Licensee MDPI, Basel, Switzerland. This article is an open access article distributed under the terms and conditions of the Creative Commons Attribution (CC BY) license (https:// creativecommons.org/licenses/by/ $4.0 /)$.
5 Department of Organic, Biochemical and Food Engineering, "Cristofor Simionescu" Faculty of Chemical Engineering and Environmental Protection, "Gheorghe Asachi" Technical University of Iasi,

700050 Iasi, Romania; alexandra-cristina.blaga@academic.tuiasi.ro

6 Department of Natural and Synthetic Polymers, "Cristofor Simionescu" Faculty of Chemical Engineering and Environmental Protection, "Gheorghe Asachi” Technical University of Iasi, 700050 Iasi, Romania; florin.ciolacu@academic.tuiasi.ro

7 Department of Environmental Engineering and Management, "Cristofor Simionescu" Faculty of Chemical Engineering and Environmental Protection, "Gheorghe Asachi" Technical University of Iasi, 700050 Iasi, Romania; icre@ch.tuiasi.ro

* Correspondence: vasilica.popescu@academic.tuiasi.ro or vpopescu65@yahoo.com

Abstract: This study may open a new way to obtain the coloration of a polymer during functionalization. Two polyacrylonitrile (PAN) polymers in the form of textile fibers (Melana and Dralon L) were subjected to functionalization treatments in order to improve the dyeing capacity. The functionalizations determined by an organo-hypervalent iodine reagent developed in situ led to fiber coloration without using dyes. $\mathrm{KIO}_{3}$ was formed in situ from the interaction of aqueous solutions of $3-9 \% \mathrm{KOH}$ with $3-9 \% \mathrm{I}_{2}$, at $120{ }^{\circ} \mathrm{C}$. The yellow-orange coloration appeared as a result of the transformations in the chemical structure of each functionalized polymer, with the formation of iodinehydrin groups. The degree of functionalization directly influenced the obtained color. The results of the Fourier Transform Infrared Spectroscopy (FTIR), Scanning Electron Microscopy (SEM), Energy Dispersive X-ray Spectroscopy (EDX), Map and Temogravimetric Analysis (TG) plus Differential Thermal (DTA) analyses indicated the presence of new functional groups, such as iodine-oxime. The X-ray diffraction (XRD) analysis confirmed the change of the crystalline/amorphous ratio in favor of the former. The new groups introduced by functionalization make it possible to dye with classes of dyes specific to these groups, but not specific to PAN fibers, thus improving their dyeing capacity.

Keywords: functionalization; polyacrilonitrile; iodinehydrin; iodine-oxime; nitrile oxides; coloring

\section{Introduction}

The functionalization of acrylic polymers is performed in order to enhance their reactivity and improve functionality, with impact on aesthetical and comfort properties. 
The color and appearance of PAN fibers depend on their tinctorial properties, which are generally satisfactory, but the dyeing process is complex and uneven shades are a common defect in PAN fiber dyeing. The lighter the desired color, the bigger the staining risk. Anionic leveling or cationic retarders are used to surpass this inconvenience but their use in dyeing recipes make the process more complicated and polluting, which is why they must be used in strictly controlled amounts.

The amount of retarder must be rigorously calculated according to a well-determined formula that involves knowing the specific indices of the cationic dyes and the acrylic polymer.

Reactive and functional PAN fibers were obtained by chemical changes of the polymer structure. Reported functionalization agents were: alkalis [1], amines such as dimethylaminopropylamine [2], ethylenediamine [3], hydrazine [4], urea or hydroxylamine [5-7], and primary or secondary acyclic aliphatic amines, diethylamine and diethylenetriamine [8] Our previous work reports PAN functionalization with alkalis, amines and eco-friendly agents such as chitosan and monochlorotriazinyl- $\beta$-cyclodextrin (MCT $\beta$-CD) [9-13].

Chemical transformations of the acrylic polymer/copolymer consist of:

a. Conversion of the ester groups of vinyl acetate (VA) into hydroxyl groups; and

b. Conversion of nitrile groups into other different functional groups (oxime, hydroxamic acid, mono/disubstituted amidines), depending on the basicity of the functionalization agent [9-13].

The chemical reactions types involved in PAN functionalization are saponification, hydrolysis, amination and $\mathrm{N}$-acylation. Generated functional groups can act as grafting sites for eco-friendly compounds [9-13].

The novelty of this study consists in the enhancement of the tinctorial capacity of PAN fibers through a sustainable dye-free functionalization. The coloration effect is the result of certain chemical modifications of the acrylic polymer, produced by functionalization.

Specialty literature shows that PAN changes color only when it is subjected to thermal treatment at above $240{ }^{\circ} \mathrm{C}$ during stabilization, as the first stage of PAN conversion into carbon fiber [14]. Color appears as result of the formation of the polyimine cycle in nitrogen [15], oxygen [16-19] or air [20], at high temperature. Stabilization in a mixture of air and ammonia, $\mathrm{NH}_{3}$ at $260{ }^{\circ} \mathrm{C}$ turns PAN's color from white to yellow and finally to black, depending on treatment severity [21].

Chemical and physical changes in the PAN structure are insignificant if the treatment is performed at temperatures below $140{ }^{\circ} \mathrm{C}$. Above this temperature, oxygen in the air can sensitize the nitrile group (polyimine cycles occur) and cause crosslinking between the macromolecular chains of PAN $[19,22]$.

Nonetheless, an adequate oxidizing agent can produce PAN coloration even at temperatures below $140{ }^{\circ} \mathrm{C}$. For example, potassium permanganate, $\mathrm{KMnO}_{4}$ acts as a catalyst for the cyclization of nitrile groups at $80^{\circ} \mathrm{C}$, when color appears in the PAN fiber [23-25].

In the present work, PAN coloration is due to functionalization with alcoholic solutions of $\mathrm{I}_{2} / \mathrm{KI}$ in alkaline medium. An iodine-potassium iodide solution alone does not stain PAN materials, but produces swelling [26,27], change of electrical conductivity and dielectric constant [26-29] and alteration of the internal structure reflected in the decrease of the crystalline/amorphous ratio [27]. These effects are related to doping, which occurs in the chemical treatment of PAN, irrespective of the iodine states of (1) vapors [28], (2) crystals dissolved in ethanol/water solution [30], or (3) an aqueous $\mathrm{I}_{2} / \mathrm{KI}$ solution in which the triiodide ion, $\mathrm{I}_{3}{ }^{-}$is generated [31,32].

The iodine-potassium iodide solution determines PAN doping and swelling. Crosslinking in swelled PAN is temperature dependent, i.e., it decreases with temperature rise $[14,33]$.

In order to avoid doping and promote PAN functionalization, we studied PAN treatment with $\mathrm{I}_{2} / \mathrm{KI}$ solution in the presence of potassium hydroxide, $\mathrm{KOH}$. It is known that $\mathrm{KOH}$ can act as a catalyst in chemical reactions taking place at high temperature [34].

A step by step technique was applied to elucidate the mechanism of chemical transformations of PAN treated with $\mathrm{I}_{2} / \mathrm{KI}$ in $\mathrm{KOH}$ solution, examining: (1) PAN functionalization 
with potassium hydroxide, $\mathrm{KOH}$; (2) PAN functionalization with $\mathrm{I}_{2} / \mathrm{KI}$ solutions; and (3) functionalization with $\mathrm{I}_{2} / \mathrm{KI}$ in $\mathrm{KOH}$ solution. For each working variant, the tinctorial properties of the acrylonitrile polymer as a function of the treatment solution composition were assessed. FTIR, EDX and XRD analyses confirmed the presence of newly formed functional groups. Treatment with $\mathrm{KOH}+\mathrm{I}_{2} / \mathrm{KI}$ for $60 \mathrm{~min}$ at $120^{\circ} \mathrm{C}$ determined PAN functionalization through the formation of iodine-oxime groups, which impart a yellow-orange color to the PAN fiber. The essential role in functionalization is played by potassium iodate, $\mathrm{KIO}_{3}$ which is generated in situ and releases molecular oxygen, $\mathrm{O}_{2}$ at $120^{\circ} \mathrm{C}$. Oxygen reacts mainly with the nitrile groups and generates oxidized unstable intermediates that readily react with iodine to form cyanohydrins of the iodine-oxime type. These are considered as pre-stabilization stages, which take place under physical (temperature $120^{\circ} \mathrm{C}$, pressure $>$ $1 \mathrm{~atm}$, aqueous medium) or chemical $\left(\mathrm{KIO}_{3} / \mathrm{O}_{2}\right.$ generated in situ) stimuli. Pre-stabilization generates in the increase of optical density and shrinkage of the treated fabric.

The novelty of this study consists in the sustainable functionalization of PAN fibers, which results in a deep and uniform yellow-orange coloration in the absence of any dyestuff. The proposed process reduces pollution and water/dye/chemical/energy demands, as compared with the conventional PAN dyeing process with cationic dyes. The proposed functionalization improves the tinctorial capacity of PAN fibers in terms of broadening the range of dyes usable for the polyacrylonitrile fibers. Thus, functionalized PAN gains an affinity for anionic dyes by means of the newly formed functional groups, which are able to establish ionic bonds with this class of dyes that is non-typical for PAN.

It is the aim of this paper to carry out PAN fiber coloration through functionalization by oxidative reactions in the presence of an environmentally sustainable organo-hypervalent iodine reagent.

\section{Materials and Methods}

\subsection{Materials}

Acrylic materials used in this study were $1 \times 1$ rib knit fabrics, in two vertical gauge values: 9.0 (sample V1) and 10 (sample V2). The commercial acrylic fibers used for the tested knits were: Melana (Rifil S.A., Savinesti, Romania) and Dralon L (Dralon GmbH Werk Ling, Lingen, Germany).

The samples were knitted on a E12 gauge (12 needles per inch) Stoll CMS-500 flat knitting machine (Karl Mayer STOLL Textilmaschinenfabrik GmbH, Reutlingen, Germany). Gauge value has an influence upon the fabric thickness in the vertical direction. The sample with gauge 9 (variant V1) has a higher vertical gauge than the sample with gauge 10 (variant V2), where the vertical gauge is the number of knitted rows per inch.

Chemical structures of the acrylic polymers from which the fibers are made are given in Figure 1.

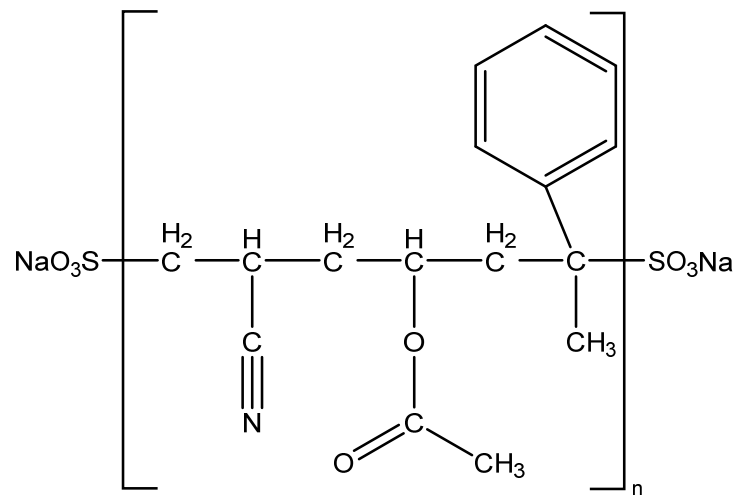

(a)

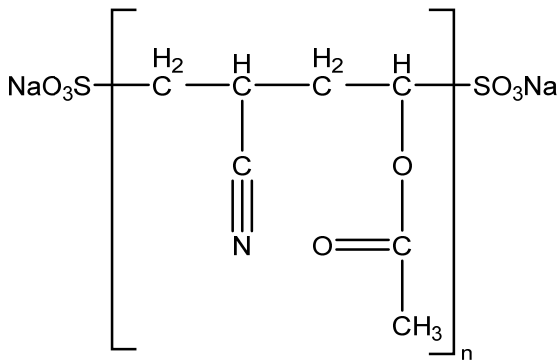

(b)

Figure 1. Chemical structures of the investigated acrylic polymers: (a) "Melana"; (b) "Dralon L". 
Other names for Dralon L: vinyl acetate/acrylonitrile copolymer and vinyl acetate/ acrylonitrile polymer.

Other names for Melana: acrylonitrile/vinyl acetate/alpha methylstyrene copolymer and acrylonitrile/vinyl acetate/alpha methylstyrene polymer. The Romanian brand Melana refers to a ternary polymer (acrylonitrile $85 \%$, vinyl acetate $10 \%$ and alphamethylstyrene $5 \%$ ) acrylic fiber obtained through radical polymerization with the redox system potassium persulfate-sodium metabisulfite.

\subsection{Reagents and Chemicals}

Potassium hydroxide $(\mathrm{KOH})$, acetic acid $\left(\mathrm{CH}_{3} \mathrm{COOH}\right)$, ethanol $\left(\mathrm{C}_{2} \mathrm{H}_{5} \mathrm{OH}\right)$, and iodinepotassium iodide $\mathrm{I}_{2} / \mathrm{KI}$ solution were purchased from Carl Roth $\mathrm{GmbH}$ (Karlsruhe, Germany).

The alkaline $\mathrm{pH}$ required for functionalization was provided by $\mathrm{KOH}\left(\mathrm{pK} \mathrm{b}_{\mathrm{b}}=0.5\right)$, as a weaker base than $\mathrm{NaOH}\left(\mathrm{pK}_{\mathrm{b}}=0.2\right)$ [35]. All chemicals were of reagent grade.

\subsection{Functionalization Experiments}

The acrylic fibers Melana and Dralon L were subjected to chemical treatments and the functionalization effects were assessed. The functionalization experiments were conducted with $\mathrm{I}_{2} / \mathrm{KI}$ solutions in the presence or absence of an alkali, namely $\mathrm{KOH}$, in accordance with the experimental protocol given in Table 1.

Table 1. Experimental protocol for the functionalization treatments.

\begin{tabular}{|c|c|c|c|}
\hline \multirow[b]{2}{*}{ Functionalization Parameters } & \multicolumn{3}{|c|}{ Concentration of Functionalization Agents } \\
\hline & $\begin{array}{c}\mathrm{KOH} \\
{[\% \text { owf }]^{*}}\end{array}$ & $\begin{array}{c}\mathrm{I}_{2} / \mathrm{KI} \\
{[\% \text { owf }]}\end{array}$ & $\begin{array}{c}\mathrm{KOH}+\mathrm{I}_{2} / \mathrm{KI} \\
{[\% \text { owf }]^{*}}\end{array}$ \\
\hline \multicolumn{4}{|c|}{ "Controlled functionalization" } \\
\hline $\begin{array}{c}\mathrm{T}=20^{\circ} \mathrm{C} \text { (room temperature) } \\
\mathrm{t}=3 \text { days, } \mathrm{M}=1: 15\end{array}$ & 9 & 9 & $9+9$ \\
\hline \multirow{3}{*}{$\mathrm{T}=120^{\circ} \mathrm{C}, \mathrm{t}=60 \mathrm{~min}, \mathrm{M}=1: 15$} & 3 & 3 & $3+3$ \\
\hline & 6 & 6 & $6+6$ \\
\hline & 9 & 9 & $9+9$ \\
\hline
\end{tabular}

$\mathrm{T}=120^{\circ} \mathrm{C}, \mathrm{t}=30 \mathrm{~min}, \mathrm{M}=1: 15$

"Severe functionalization"

Due to excess of chemical reagents

Knit fabric variants: V1 and V2

\footnotetext{
$* \%$ owf $=$ percent on weight of fabric.
}

Functionalization treatments were conducted in sealed stainless-steel vessels at two different temperatures: room temperature $\left(20{ }^{\circ} \mathrm{C}\right)$ and $120{ }^{\circ} \mathrm{C}$, respectively. Reagent amounts were calculated as percent on weight of fabric samples, in the range 3-9\%; the liquor ratio $(\mathrm{M})$ was 1:15.

Functionalization treatments were followed by rinsing with warm and cold water, neutralization with acetic acid, wringing and air drying.

\subsection{Analysis of the Functionalized Fibers}

\subsubsection{FTIR Analysis}

The FTIR spectra of the acrylic materials were recorded on a Bruker Optics equipment(Bruker Optik GmbH, Ettlingen, Germany), comprising a TENSOR 27 FTIR spectrophotometer(Bruker Optik GmbH, Ettlingen, Germany), adequate mainly for near-IR, coupled with a HYPERION 1000 microscope equipped with a standard $15 \times$ objective. The standard DLaTGS detector works in the $7500-370 \mathrm{~cm}^{-1}$ spectral range, with a resolution of $4 \mathrm{~cm}^{-1}$. The TENSOR 27 spectrophotometer is equipped with a He-Ne laser that operates at a wavelength of $633 \mathrm{~nm}$ and an output power of $1 \mathrm{~mW}$, and presents a ROCKSOLID alignment of the interferometer. TENSOR 27 was assisted by OPUS software, which allowed for the acquisition of interactive video data. 
The liquid nitrogen-cooled MCT detector covered the spectral range $600-7500 \mathrm{~cm}^{-1}$; measured aria was optimized to $250 \mu \mathrm{m}$, but can reach a minimum of $20 \mu \mathrm{m}$.

\subsubsection{XRD Analysis}

XRD analysis was performed on an X'PERT PRO MRD X-ray generator (PANalytical, Almelo, The Netherlands) with the following characteristics: tube voltage $=35 \mathrm{kV}$, tube current $=20 \mathrm{~mA}$., vacuum pressure $=(1 / 2) \mathrm{mbar}$, slit width $=80 \mu \mathrm{m}$, counter slit width $=250 \mu \mathrm{m}$, wavelength of $\mathrm{Cu} \mathrm{k} \alpha$ radiation $\lambda=1.5418 \mathrm{~A}^{0}$, the counter- sample distance $\mathrm{A}=20 \mathrm{~cm}$, capillary diameter $(\mathrm{d})=1 \mathrm{~mm}$, working temperature $22.5 \pm 0.5^{\circ} \mathrm{C}$. Monochromatic $\mathrm{Cu} k \alpha\left(\lambda=1.54 \mathrm{~A}^{0}\right)$ radiation was obtained with a nickel filter of $10 \mu \mathrm{m}$ thickness, used to irradiate acrylic fibers packed in a Mark capillary tube(Capillary Tube Supplies Ltd., Rose Cottage, United Kingdom) of 1 mm diameter.

\subsubsection{SEM + Map + EDX}

The SEM plus Map plus EDX analyses were performed on an SEM microscope model VEGA II LSH (TESCAN S.R.O., Brno, Czech Republic) coupled with a 3rd generation EDX detector, model QUANTAX QX2 (BRUKER Optics, Ettlingen, Germany).

The main features of the microscope were a tungsten heated cathode, resolution $3 \mathrm{~nm}$ at $30 \mathrm{kV}$, scanning speed from $200 \mathrm{~ns}$ to $10 \mathrm{~ms}$ per pixel, magnification $13-1.000 .000 \times$ in resolution mode at $30 \mathrm{kV}$, accelerating voltage $200 \mathrm{~V}$ to $30 \mathrm{kV}$ and working pressure below $1 \times 10^{-2} \mathrm{~Pa}$. The XFlash EDX detector(Bruker Optik GmbH, Ettlingen, Germany), used for qualitative and quantitative analysis is 10 times faster than conventional $\mathrm{Si}(\mathrm{Li})$ detectors.

The SEM-EDX coupling allows, at the same time, microphotogram acquisition, surface imaging with atom mapping, and determination of the elemental composition (in mass or molar fractions) of a microstructure or a selected area of a sample.

\subsubsection{Thermal Resistance/Thermal Conductivity}

Thermal resistances of the acrylic fabrics was determined on a Permetest Sensora device(Sensora Instruments \& Consulting, Liberec, Czech Republic).

\subsubsection{TG and DTA Thermal Analysis/Thermogravimetry}

Thermogravimetric analysis was performed on a computer-aided Linseis STA PT-1600 (Linseis Messgeraete $\mathrm{GmbH}$, Selb, Germany) thermobalance with simultaneous recording of the thermogravimetric curves. The working conditions were heating rate $10^{\circ} \mathrm{C} / \mathrm{min}$ in a dynamic air atmosphere and gas flow of $50 \mathrm{~mL} / \mathrm{min}$, maximum temperature $800^{\circ} \mathrm{C}$, samples weighed $50 \mathrm{mg}$, as measured on an electronic balance model PARTNER AS220/C/2.

\subsubsection{Color Measurements}

Colorimetric measurements CIELab and color intensity (K/S), were performed on functionalized samples, and on functionalized and dyed samples. To prove the presence of functional groups in the polymer chain, dyeing was performed with non-typical dyes, such as acid dyes.

Color was quantified based on the CIELab color model, using the experimental determination of $\mathrm{L}^{*}, \mathrm{a} * \mathrm{~b} * \mathrm{C} *$ and $\mathrm{h} *$ values on a Datacolor Sprectroflash SF300 spectrophotometer(Datacolor, Lucerne, Switzerland). The significance of coloristic measurements are as follows: $\mathrm{L}^{*}$ stands for luminosity; $\mathrm{a}$ * and $\mathrm{b}$ * are the position of color on the red-green and yellow-blue coordinates; $C *$ is color saturation and $\mathrm{h} *$ is color shade.

Color intensity of dyed fabric samples, K/S was calculated with the Kubelka-Munk equation:

$$
\mathrm{K} / \mathrm{S}=\left[(1-\mathrm{R})^{2}\right] / 2 \mathrm{R}
$$

where the reflectance of the dyed fabrics, R [\%] was measured on the same Datacolor Sprectroflash SF300 spectrophotometer (Datacolor, Lucerne, Switzerland). 


\subsubsection{Statistical Analysis}

Experimental values of electrical conductivity and color intensity were subjected to statistical processing. Error of the mean, standard deviation (SD) and the coefficient of variation $(\mathrm{CV})$ were calculated in Matlab and indicated on the related figures.

\section{Results and Discussion}

\subsection{Mechanism of PAN Functionalization}

The attempt to elucidate the mechanism of acrylic polymer functionalization was based on a thorough documentation of previous work regarding the behavior of acrylic polymers in alkaline media and their interaction with iodine or $\mathrm{I}_{2} / \mathrm{KI}$ solutions.

Treating PAN fibers with $2.5 \% \mathrm{NaOH}$ aqueous solution at $100{ }^{\circ} \mathrm{C}$ produces functionalization due to the generation of amide or carboxyl functional groups, with no color change [9-11]. The development of a yellow-orange color was noticed when functionalization was carried out with amine compounds such as hydroxylamines [9], which converted the nitrile groups into imine groups, more precisely into oxime groups $(\mathrm{HO}-\mathrm{NH}-\mathrm{C}=\mathrm{N})$ [13].

Yue et al. [34] studied the effect of alkalis upon the nitrile group in acrylonitrile and concluded that alkaline compounds act as catalysts for the conversion of acrylonitrile into acrylic acid by hydrothermal reaction $\left(300^{\circ} \mathrm{C}\right)$ and that potassium hydroxide, $\mathrm{KOH}$ is the most effective alkaline agent. Studies on the interaction between PAN and iodine from alcoholic solutions of $\mathrm{I}_{2} / \mathrm{KI}$ put in evidence polyacrylonitrile doping, which is favored by temperature decrease $[26-29,36]$. Other authors $[37,38]$ state that iodine penetrates the polymer crystalline zones and forms a complex with the polyacrylic chain due to its ability to interact with the nitrile group.

In fact, weak physical electrostatic interaction between the $\mathrm{K}^{+}$ion and $\mathrm{C}=\mathrm{O} / \mathrm{C} \equiv \mathrm{N}$ is more likely. The newly formed structure has a higher ionic conductivity, which is dependent on temperature and KI concentration [38].

Other studies have shown that the amorphous region is the host of polymer matrix doping with iodine salt $[30,38]$, but iodine is easily washed off with water or acetone. Doping determines alteration of the crystalline:amorphous regions ratio, polymer swelling [26,27], and increase of electrical conductivity [28,30,36-38].

In alcoholic solutions of $\mathrm{I}_{2} / \mathrm{KI}$, iodine is present as a triiodide ion, $\mathrm{I}_{3}{ }^{-}$. The presence of triiodide in the PAN matrix can be easily detected by the starch test, when the appearance of a blue-black color proves formation of the iodine-starch complex. When only iodide is present in the polymer, no color change will be noticed, because iodide does not react with starch $[31,32]$.

In this study, the two acrylonitrile polymers acquired new functional groups consequent to treatment with potassium hydroxide solutions, in the presence or absence of alcoholic $\mathrm{I}_{2} / \mathrm{KI}$ solutions. When treatment was performed with $\mathrm{KOH}$ alone, two kinds of functional groups are generated:

1. Hydroxyl groups, $-\mathrm{OH}$ due to the saponification of acetate groups of vinyl acetate (VA), simultaneously with acetic acid, $\mathrm{CH}_{3} \mathrm{COOH}$ release; and

2. Carboxyl groups resulting from the hydrolysis of nitrile groups, via hydroxamic acid/amide as intermediates.

Our previous works have shown that PAN fiber functionalization with sodium hydroxide did not alter fiber color and generated acidic functional groups by fast hydrolysis because $\mathrm{NaOH}$ is a strong base $\left(\mathrm{pk}_{\mathrm{b}}=0.2\right)$. In the present work, alkaline $\mathrm{pH}$ is provided by $\mathrm{KOH}$, a weaker base $\left(\mathrm{pk}_{\mathrm{b}}=0.5\right)$, the hydrolysis reaction rate is lower, and the reaction mechanism involves the formation of hydroxamic acid instead of amide as an intermediate product. The final product is the acidic group $(-\mathrm{COOH})$ together with hydroxylamine, and $\mathrm{NH}_{2}-\mathrm{OH}$ as secondary product. Hydroxylamine has affinity for nitrile groups (not in- 
volved in functionalization) and interacts with these groups to generate oxime/amidoxime groups [39]. Possible reactions are as follows:

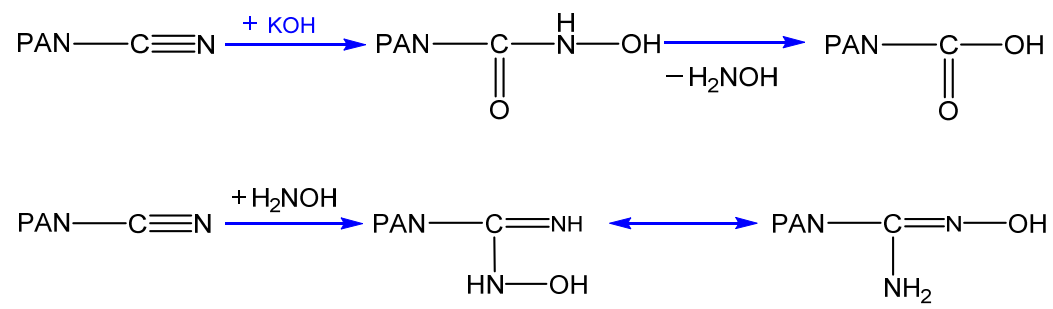

When PAN is treated with $\mathrm{KOH}$ and iodine in ethanol solution at $120^{\circ} \mathrm{C}$, potassium iodate and $\mathrm{KIO}_{3}$ generated in situ due to high temperature decompose and release molecular oxygen, $\mathrm{O}_{2}$ (Equations (5) and (6)); both $\mathrm{KIO}_{3}$ and $\mathrm{O}_{2}$ can oxidise the oxime/amidoxime/nitrile groups to nitrile oxide [40].

Generally, oxidation is accompanied by color change of PAN fibers, which is attributed to the pre-oxidation of the nitrile groups of the polymer. The pre-oxidation degree can be assessed by means of the extent of oxidation reaction (EOR) index, given by the formula: $\mathrm{EOR}=\left[\mathrm{I}_{1600} /\left(\mathrm{I}_{\mathrm{CN}}+\mathrm{I}_{1600}\right)\right.$, where $\mathrm{I}_{1600}$ stands for the absorbance intensity from $1600 \mathrm{~cm}^{-1}$ and $\mathrm{I}_{\mathrm{CN}}$ stands for the absorbance intensity of $\mathrm{CN}$ groups, in the IR domain. Extreme values of EOR are 0 and 1: when EOR $=0$ none of the nitrile groups are pre-oxidised, and when $\mathrm{EOR}=1$, all nitrile groups in the polymer underwent oxidation [41].

Besides the pre-oxidation effect, when temperature exceeds $140^{\circ} \mathrm{C}$, oxygen may play an essential role in the cyclization process by two opposite effects: initiation of active site formation that is responsible for cyclization, and cyclization inhibition by rise of activation energy. When working temperature was lower than $140{ }^{\circ} \mathrm{C}$, FTIR analysis of the treated acrylic polymer did not confirm the presence of cycles [42].

The hypervalent potassium iodate, $\mathrm{KIO}_{3}$ is a versatile and environmentally friendly reagent that can be used in different oxidative transformations [40].

Potassium iodate generated in situ is a hypervalent $\mathrm{I}(\mathrm{v})$ compound with high oxidizing strength and ability to convert amidoxime into nitril oxide, a nonisolable compound. Nitrile oxides are efficient 1,3-dipolar reactants in intra- or intermolecular cycloadditions [40,42]. Nitrile oxides are unstable and extremely reactive; they dimerize to form furoxans, hydrolyze to form $\mathrm{N}$-hydroxyamides (hydroxamic acid) as final products, or interact with iodine in the reaction mixture. These functionalization reactions determine the permanent coloration of acrylic fibers in yellow-orange shades [40].

Chemical reactions involved in the conversion of nitrile groups of PAN chains in the above-mentioned functional groups are as follows:

(a) Conversion to amidoxime groups placed on the polymeric chain (Equations (2) and (3)), and in situ obtaining of potassium iodate at $120^{\circ} \mathrm{C}$ (Equations (4)-(8)):

$$
\underset{6 \mathrm{KOOH}+3 \mathrm{I}_{2} \stackrel{\left[\mathrm{t}=120^{\circ} \mathrm{C}\right]}{\longrightarrow}-3 \mathrm{~K}_{2} \stackrel{\left[\mathrm{t}=120^{\circ} \mathrm{C}\right]}{\longrightarrow} \mathrm{KI}+3 / 2 \mathrm{O}_{2}+5 \mathrm{KI}+3 \mathrm{H}_{2} \mathrm{O}}{\longrightarrow} 6 \mathrm{KI}+3 / 2 \mathrm{O}_{2}+3 \mathrm{H}_{2} \mathrm{O}
$$

In reaction (4), $I_{2}$ is both an oxidizing and a reducing agent (disproportionation/ dismutation of elemental iodine), as follows:

$$
6 \mathrm{KOH}+3 \mathrm{I}_{2}^{0} \longrightarrow \mathrm{KI}^{5} \mathrm{O}_{3}+5 \mathrm{KI}^{-1}+3 \mathrm{H}_{2} \mathrm{O}
$$




$$
\begin{aligned}
& 5 \mathrm{I}^{0}+5 \mathrm{e}^{-} \longrightarrow 5 \mathrm{I}^{-1} \quad \text { (reduction) } \\
& \mathrm{I}^{0}-5 \mathrm{e}^{-} \longrightarrow \mathrm{I}^{5} \quad \text { (oxidation) }
\end{aligned}
$$

(b) Generation of nitrile oxides, through amidoxime oxidation by $\mathrm{KIO}_{3} / \mathrm{O}_{2}$ [43-45] (Equation (9)):

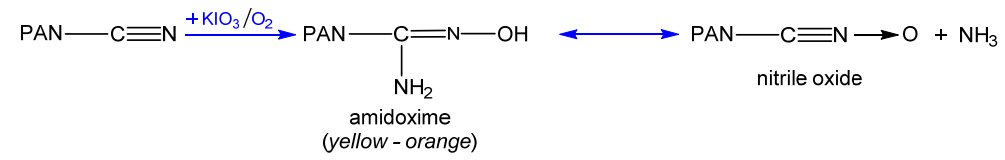

Oxidized nitrile groups are unstable intermediates (Equation (10)) [46-48] and readily turn into ionic forms that interact both with the $\mathrm{I}^{-}$ion (derived from KI dissociation) and the $\mathrm{H}^{+}$ion present in the reaction medium to generate polyacrylonitrile functionalized with iodine-oxime groups, PAN-C(I)=N-OH (Equation (11).

$\mathrm{PAN}-\mathrm{C} \equiv \mathrm{N} \stackrel{\oplus}{=} \mathrm{O}^{\ominus} \longleftrightarrow \mathrm{PAN}-\mathrm{C}=\mathrm{P}=\mathrm{N} \stackrel{\mathrm{O}}{=} \mathrm{O} \longleftrightarrow \mathrm{PAN}-\mathrm{C}^{\oplus}=\mathrm{N}-\mathrm{O}^{\ominus} \longleftrightarrow \mathrm{PAN}-\mathrm{C}=\mathrm{\ominus}=\mathrm{N}-\mathrm{O}^{\oplus} \longleftrightarrow \mathrm{PAN}-\ddot{\mathrm{C}} \mathrm{H}-\mathrm{N}=\mathrm{O}$

Nitrile oxides are mild oxidizing agents that release iodine from an acidified solution of KI [47]. The presence of both $\mathrm{I}^{-}$and $\mathrm{H}^{+}$ions makes possible the emergence of cyanohydrin of the iodinehydrin type [47]. Iodine present in iodinehydrin hinders dimerization and cyclization, having a stabilization effect [47].

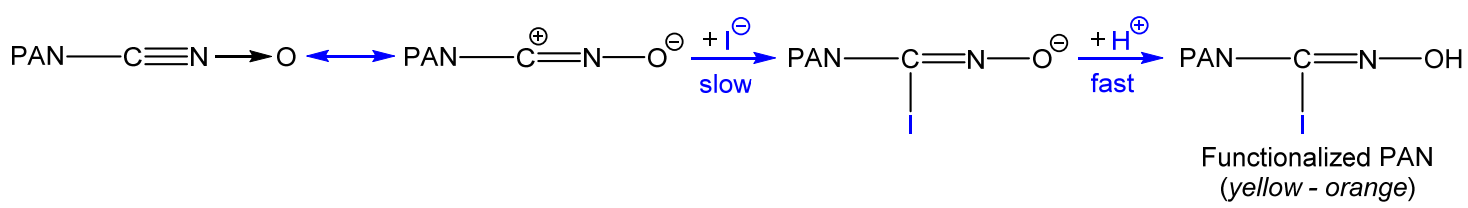

The $\mathrm{pH}$ measurement of treating solutions before and after functionalization showed $\mathrm{pH}$ decreased during treatment (Table 2), which proved the generation of acidic compounds during the reaction. Hydrogen iodide, HI, resulted from the reaction between water and $\mathrm{KI}$, and acetic acid, $\mathrm{CH}_{3} \mathrm{COOH}$, derived from the saponification of the acetate group present in vinyl acetate.

\begin{tabular}{|c|c|c|c|c|c|}
\hline \multirow[b]{2}{*}{ Sample } & \multirow[b]{2}{*}{ Functionalization Treatment } & \multicolumn{2}{|c|}{ Before Functionalization } & \multicolumn{2}{|c|}{ After Functionalization } \\
\hline & & $\mathrm{pH}$ & $\begin{array}{c}\text { Conductibility } \\
(\mu \mathrm{S} / \mathrm{cm})\end{array}$ & $\mathrm{pH}$ & $\begin{array}{c}\text { Conductibility } \\
(\mu \mathrm{S} / \mathrm{cm})\end{array}$ \\
\hline \multirow{3}{*}{ Dralon L } & $6 \% \mathrm{KOH}$ & 12.27 & 13,475 & 11.37 & 10,520 \\
\hline & $6 \% \mathrm{KI}$ & 7.9 & 3160 & 7.26 & 3146 \\
\hline & $6 \% \mathrm{KOH}+6 \% \mathrm{I}_{2} / \mathrm{KI}$ & 6.65 & 1828 & 4.65 & 2166 \\
\hline \multirow{3}{*}{ Melana } & $6 \% \mathrm{KOH}$ & 11.42 & 13,430 & 11.28 & 12,476 \\
\hline & $6 \% \mathrm{KI}$ & 7.63 & 3166 & 6.31 & 3320 \\
\hline & $6 \% \mathrm{KOH}+6 \% \mathrm{I}_{2} / \mathrm{KI}$ & 6.64 & 1753 & 5.25 & 2254 \\
\hline
\end{tabular}

Table 2. $\mathrm{pH}$ and conductivity of treating liquors before and after functionalization.

The iodine ion, $\mathrm{I}^{-}$originates from the dissociation of KI, confirmed by solution conductivity before and after functionalization, increased the conductibility of the residual liquor after functionalization with $\mathrm{KOH}+\mathrm{I}_{2}$ due to the increase of the $\mathrm{K}^{+}$concentration, which demonstrates that potassium iodide is not involved in the PAN/KI complex formation [30]. According to some authors, formation of this complex indicates PAN doping with iodine [30]. 
The triiodide ion, $\mathrm{I}_{3}{ }^{-}$is present in aqueous solutions of $\mathrm{I}_{2} / \mathrm{KI}$, but is not detected when the starch test is applied on the functionalized acrylic polymers, and the characteristic blue-black coloration indicating iodine presence does not appear [31,32].

PAN functionalization was confirmed both by the FTIR spectra and the colorimetric measurements performed on treated yellow-orange samples.

\subsection{FTIR Results for the Polyacrylonitrile Materials}

Comparative examination of FTIR spectra of Melana and Dralon L before and after treatment confirmed functionalization, by the presence of carboxyl, of the oxime and hydroxyl groups in the treated samples (Figures 2 and 3). These functional groups emerged as a result of chemical modifications of the nitrile group of the AN monomer and the acetate groups of the VA monomer, respectively. The nitrile group has an intense adsorption band at $2242 \mathrm{~cm}^{-1}$, while the aliphatic nitrile oxide intermediates have two adsorption bands at around $2330 \mathrm{~cm}^{-1}(\mathrm{C}=\mathrm{N}$ stretching $)$ and around $1370 \mathrm{~cm}^{-1}(-\mathrm{N}=\mathrm{O}$ stretching $)$. The $\mathrm{C}=\mathrm{N}$ stretching band is preferred for the identification of monomeric nitrile oxides [47].

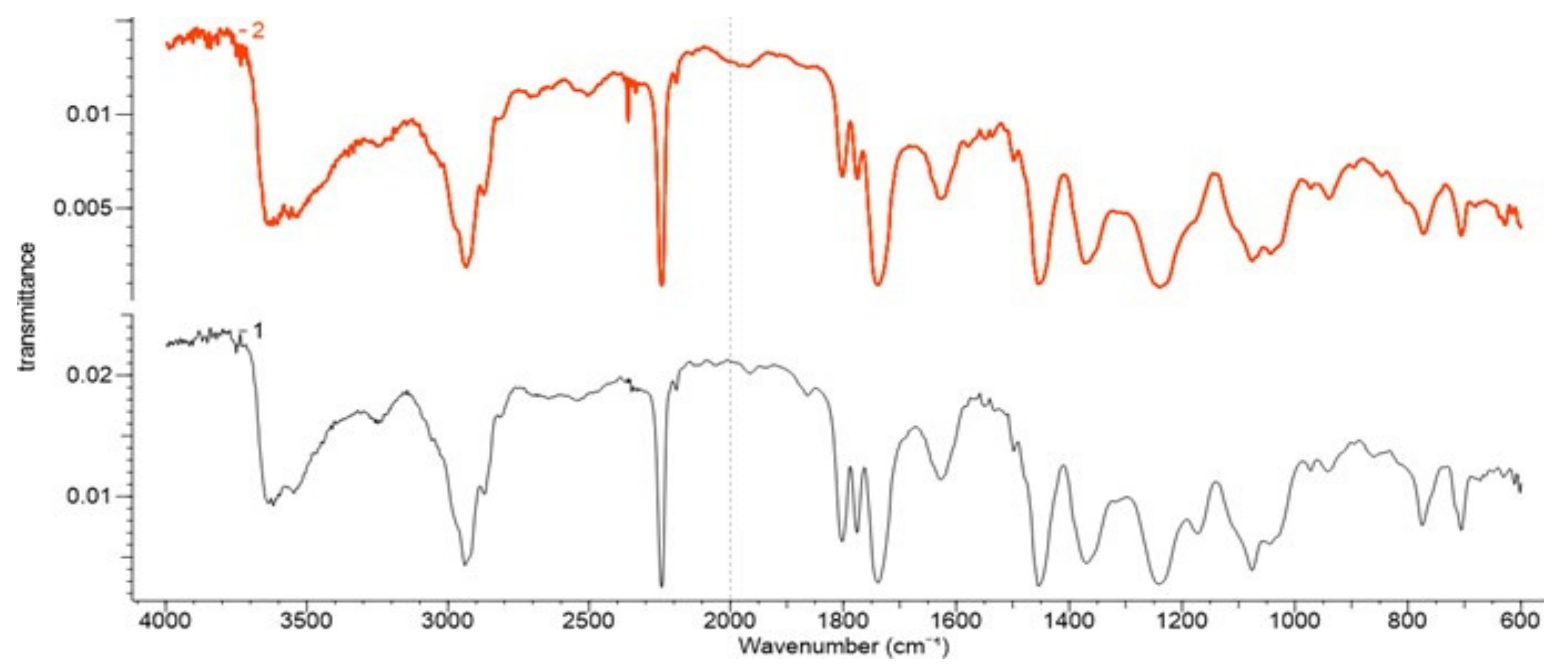

Figure 2. FTIR spectra of Melana: (1) pristine/untreated and (2) after functionalization.

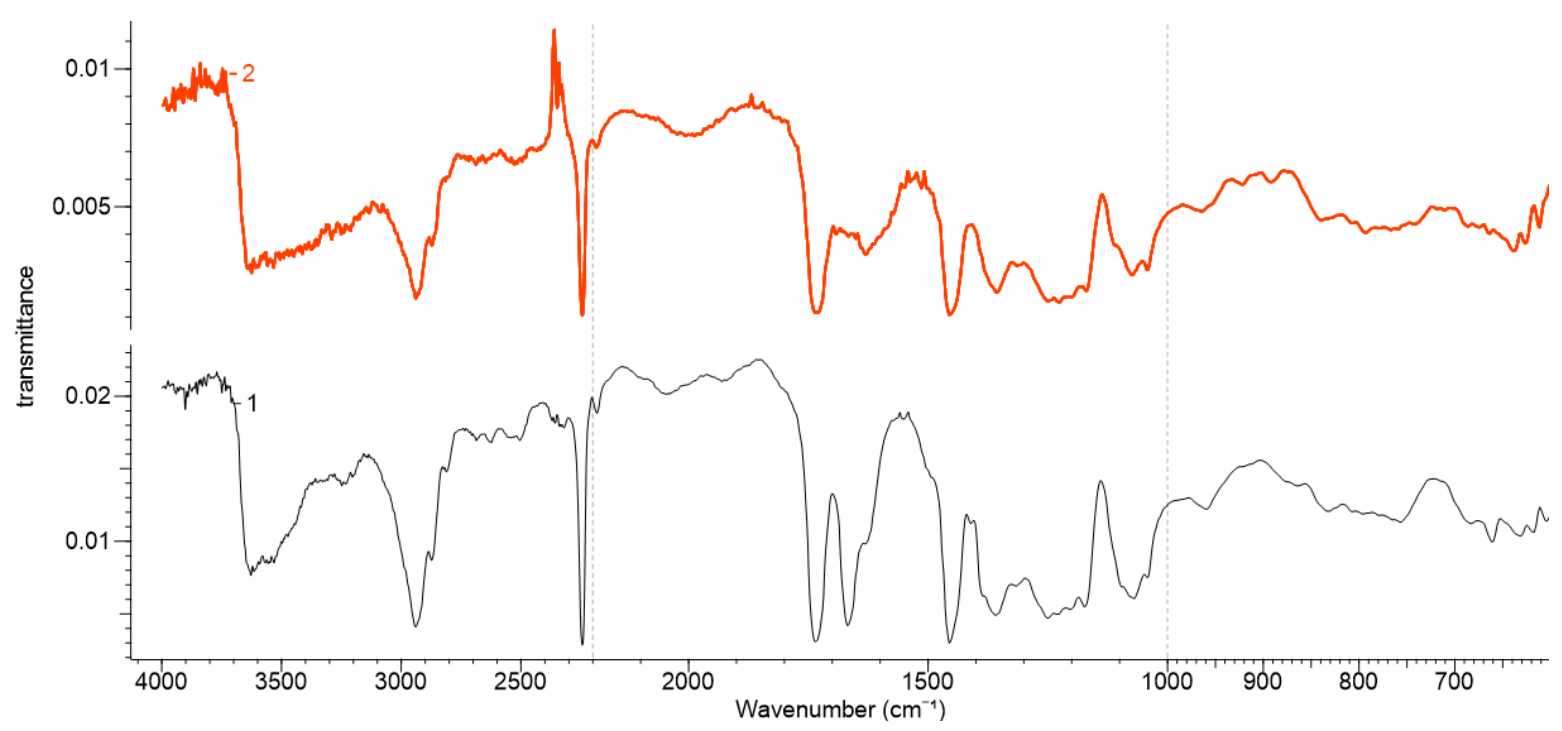

Figure 3. FTIR spectra of Dralon L: pristine (1) and (2) after functionalization. 
Carboxyl groups $(-\mathrm{COOH})$ generated by the alkaline hydrolysis of a part of the nitrile groups ere confirmed by the increase of $1746-1735 \mathrm{~cm}^{-1}$ ( $\mathrm{C}=\mathrm{O}$ stretching) and $1249-1240 \mathrm{~cm}^{-1}$ (C-O stretching) peak intensities. Newly formed carboxyl groups increase the oxygen content of the functionalized acrylic polymers, even if the saponification of ester groups of VA with formation of hydroxyl groups $(-\mathrm{OH})$ is prone to deprive the polymer of oxygen.

The presence of oxime groups $(\mathrm{C}=\mathrm{N}-\mathrm{OH})$ in Melana functionalized with iodine-oxime groups $(\mathrm{C}(\mathrm{I})=\mathrm{N}-\mathrm{OH})$ as confirmed by the three characteristic IR bands: $3639 \mathrm{~cm}^{-1}(\mathrm{O}-\mathrm{H})$, $1628 \mathrm{~cm}^{-1}(\mathrm{C}=\mathrm{N})$ and $939 \mathrm{~cm}^{-1}(\mathrm{~N}-\mathrm{O})$. The peak at $628 \mathrm{~cm}^{-1}$ was assigned to iodine from the iodinehydrin group. Adsorbtion bands at $3539 \mathrm{~cm}^{-1}(\mathrm{O}-\mathrm{H})$ and $1076 \mathrm{~cm}^{-1}(\mathrm{C}-\mathrm{O}$ stretch (s)) were assigned to hydroxyl groups derived from saponification of acetate groups of VA.

Dralon $\mathrm{L}$ functionalization with $\mathrm{KOH}+\mathrm{I}_{2} / \mathrm{KI}$ was proven by the presence of adsorption bands characteristic to the newly formed functional groups, namely iodineoxime/iodinehydrin, $(\mathrm{C}(\mathrm{I})=\mathrm{N}-\mathrm{OH})$, and hydroxyl $(-\mathrm{OH})$. New adsorption bands in the functionalized Dralon $\mathrm{L}$ were compared with the untreated material and assigned to oximes $\left(3633 \mathrm{~cm}^{-1}(\mathrm{O}-\mathrm{H}), 1631 \mathrm{~cm}^{-1}(\mathrm{C}=\mathrm{N})\right.$ and $\left.930 \mathrm{~cm}^{-1}(\mathrm{~N}-\mathrm{O})\right)$. The adsorption band at $638 \mathrm{~cm}^{-1}$ was attributed to iodine in Dralon $\mathrm{L}$ functionalized with iodine-oxime groups. The hydroxyl groups generated by the saponification of ester groups of VA had adsorption bands at $3537 \mathrm{~cm}^{-1}(\mathrm{O}-\mathrm{H})$ and $1074-1070 \mathrm{~cm}^{-1}$ (C-O stretch (s)).

The absorption bands of existing functional groups in untreated PAN fibers [9-12] and those of newly born functional groups due to functionalization with $\mathrm{KIO}_{3}$ [38] are given in Table 3.

Table 3. FTIR adsorption bands of PAN fibers before and after functionalization with $\mathrm{KIO}_{3}$.

\begin{tabular}{|c|c|c|c|c|c|}
\hline Bond & Mode & $\begin{array}{l}\text { Untreated } \\
\text { Dralon L }\end{array}$ & Dralon L & $\begin{array}{l}\text { Untreated } \\
\text { Melana }\end{array}$ & $\begin{array}{c}\text { Functionalized } \\
\text { Melana }\end{array}$ \\
\hline $\mathrm{O}-\mathrm{H}$ & Stretching & $3627-3531$ & $3625-3137$ & $3637-3546$ & $3623-3531$ \\
\hline $\mathrm{C}-\mathrm{H}$ & Stretching & $2941-2873$ & $2939-2875$ & $2941-2869$ & $2937-2873$ \\
\hline $\mathrm{C} \equiv \mathrm{N}$ & Stretching & 2242 & 2244 & 2242 & 2242 \\
\hline $\mathrm{C}=\mathrm{O}$ & Symmetric Stretching & 1735 & 1735 & 1746 & 1746 \\
\hline $\mathrm{C}=\mathrm{N}$ & Stretching & - & 1629 & 1627 & 1625 \\
\hline $\mathrm{CH}_{2}$ & Symmetric Deformation & 1456 & 1456 & $1498-1454$ & $1498-1454$ \\
\hline $\mathrm{CH}_{3}$ & Symmetric Deformation & 1359 & 1357 & 1369 & 1371 \\
\hline $\begin{array}{c}\mathrm{C}-\mathrm{O}-\mathrm{C} \\
\mathrm{C}-\mathrm{H}\end{array}$ & $\begin{array}{l}\text { Stretching } \\
\text { Wagging }\end{array}$ & 1249 & 1226 & 1242 & 1240 \\
\hline $\mathrm{C}-\mathrm{OH}$ & Stretching & 1070 & 1074 & 1076 & 1076 \\
\hline $\mathrm{N}-\mathrm{O}$ & From oxime & - & 930 & - & 939 \\
\hline $\mathrm{C}-\mathrm{H}$ & $\mathrm{C}-\mathrm{H}$ rocking of pure $\mathrm{PAN}$ & 839 & 841 & 863 & 901 \\
\hline for $\mathrm{KIO}_{3}$ & - & 761 & - & 773 & \\
\hline & C-I Stretch & - & 638 & - & 628 \\
\hline
\end{tabular}

The adsorption band at about $762 \mathrm{~cm}^{-1}$ was assigned to potassium iodate, $\mathrm{KIO}_{3}$, while spectra modification due to functionalization could be observed at $761 \mathrm{~cm}^{-1}$ in Dralon $\mathrm{L}$ and at $773 \mathrm{~cm}^{-1}$ in Melana (Figures 2 and 3).

According to the literature, KI determines a frequency shift of the $\mathrm{C}-\mathrm{H}$ rocking vibration [38]. Such vibrational shifts were detected in the studied PAN polymers: from $839 \mathrm{~cm}^{-1}$ (Dralon L) and $863 \mathrm{~cm}^{-1}$ (Melana), to $841 \mathrm{~cm}^{-1}$ and $901 \mathrm{~cm}^{-1}$, respectively.

The comparative assessment of FTIR spectra of pristine and functionalized PAN fibers put in evidence: 
- The decrease in the peak intensity of $\mathrm{CH}_{3}$ (C-H stretching), due to splitting of acetate group of VA and formation of $\mathrm{OH}$ group; and

- The decrease in the peak intensity of nitrile group (CN stretching), due to conversion into carboxyl and oxime groups.

The extent of oxidation reaction, EOR was calculated based on:

1. The intensity of peaks at around $1600 \mathrm{~cm}^{-1}$, associated with $\mathrm{C}=\mathrm{N}$ stretching (namely $1625 \mathrm{~cm}^{-1}$ in Melana or $1629 \mathrm{~cm}^{-1}$ in Dralon L); and

2. The intensity of peaks associated with the nitrile groups.

Values of EOR after the $\mathrm{KOH}$ treatment were 0.4275 for Melana and 0.4832 for Dralon L. Fabrics functionalized with $\mathrm{KOH}+\mathrm{I}_{2} / \mathrm{KI}$ had very closed values of EOR: 0.425 for Melana and 0.455 for Dralon L. The EOR values indicated the contribution of the oxidation reaction in the functionalization of each acrylic polymer studied herein.

\subsection{XRD Results Interpretation}

Literature data state that treating PAN with $\mathrm{I}_{2} / \mathrm{KI}$ results in a decrease of the polymer's degree of crystallinity. The presence of $\mathrm{KI}$ in the polymer lowers the transition temperature, $\mathrm{T}_{\mathrm{g}}$ from $90^{\circ} \mathrm{C}$ to $71{ }^{\circ} \mathrm{C}$ and the melting point from $300{ }^{\circ} \mathrm{C}$ to $256^{\circ} \mathrm{C}$. The drop in the $\mathrm{T}_{\mathrm{g}}$ value indicates an increase of the extent of the amorphous regions of the polymer [38].

Treating acrylic polymers with $\mathrm{I}_{2} / \mathrm{KI}$ in the presence of $\mathrm{KIO}_{3}\left(\right.$ and $\mathrm{O}_{2}$ ) generated in situ results in the sensitization of nitrile groups by oxidation and the increase of crystallinity.

Melana and Dralon L are semi-crystalline acrylic polymers, which means that both amorphous and crystalline regions are present in the polymer chain (Figures 4-6). XRD patterns of these polymers were extensively studied in our previous work [49].

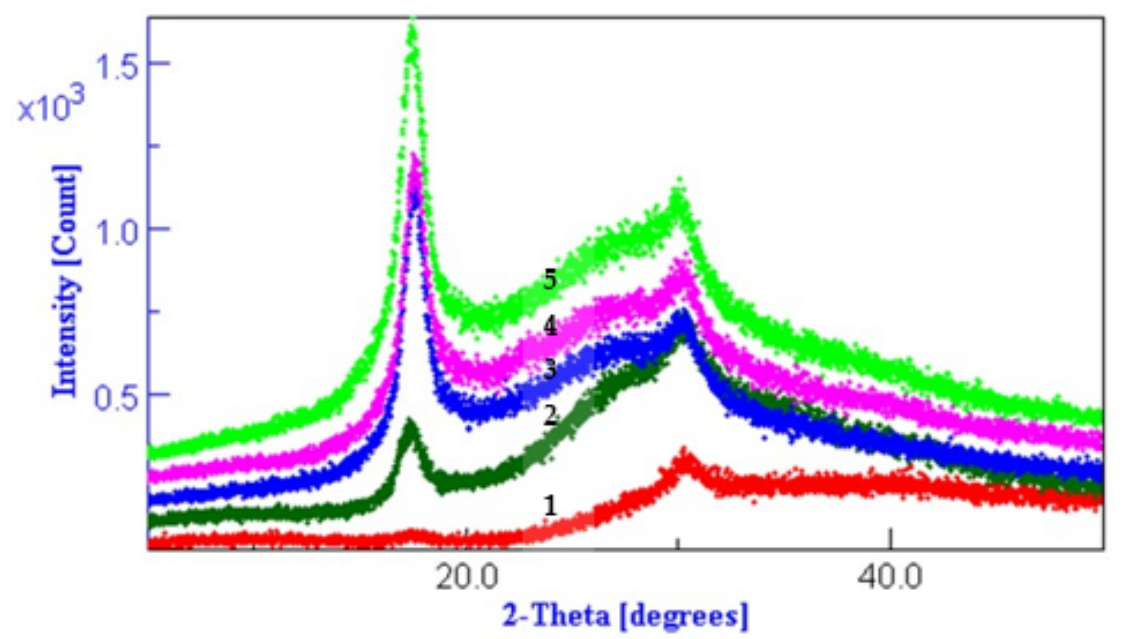

Figure 4. Diffractograms of Dralon L treated at $120{ }^{\circ} \mathrm{C}$ for $60 \mathrm{~min}$ : (1) control sample, (2) boiled in water, (3) $6 \% \mathrm{KOH}$, (4) $6 \% \mathrm{KOH}$ plus $6 \% \mathrm{I}_{2} / \mathrm{KI}$, and (5) $6 \% \mathrm{KOH}$ plus $6 \%$ iodine.

Hydrothermal or chemical treatment in aqueous media determine the increase of Melana and Dralon L polymer crystallinity (Figures 4-6). This behavior is in accordance with literature data, which indicate an increase of the polymer internal order when the working temperature is higher than $100{ }^{\circ} \mathrm{C}$, such in case of microwave-assisted [49] or thermal treatment [50-52].

The working temperature of $120^{\circ} \mathrm{C}$ was responsible for a certain degree of pre-fixation of acrylonitrile polymers, confirmed by the increase of peaks from positions 17 and 29 (2-Theta).

Lack of swelling and disturbance of the PAN fiber internal structure proves that iodine doping did not occur. Iodine attaches to the $\mathrm{C}$ atom of the oxime group to form the iodine-oxime/iodinehydrin bond, as proven by the FTIR analysis. 


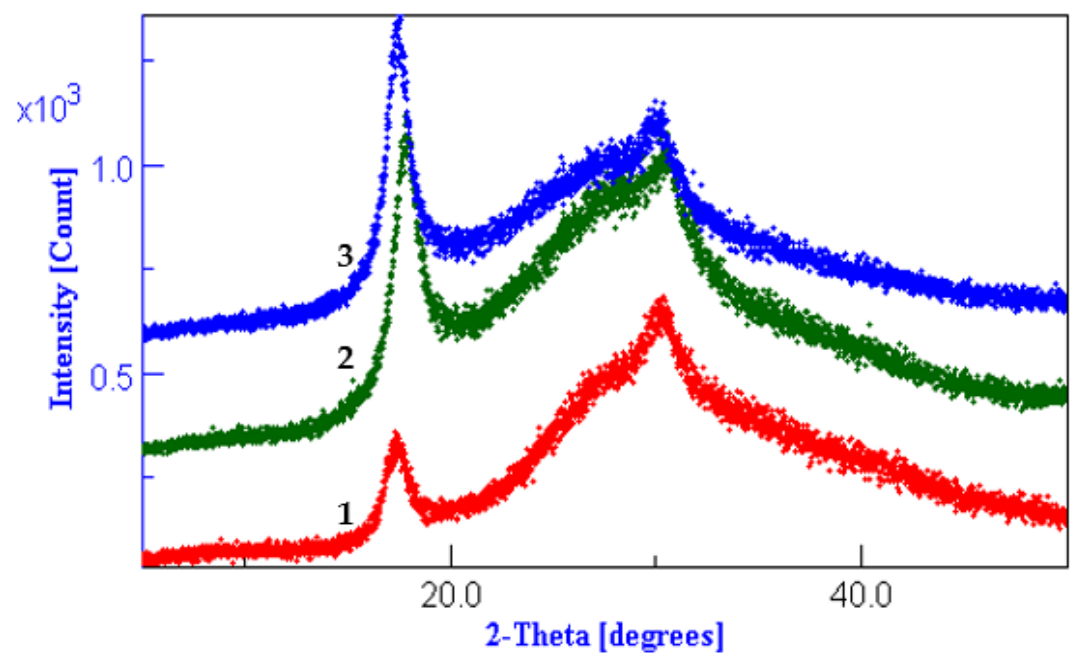

Figure 5. Diffractograms of Dralon L treated at $120^{\circ} \mathrm{C}$ for $30 \mathrm{~min}$ with excess of reagents: (1) control sample, (2) sample V1 with $\mathrm{KOH}$ plus iodine, and (3) sample V2 with $\mathrm{KOH}$ plus iodine.

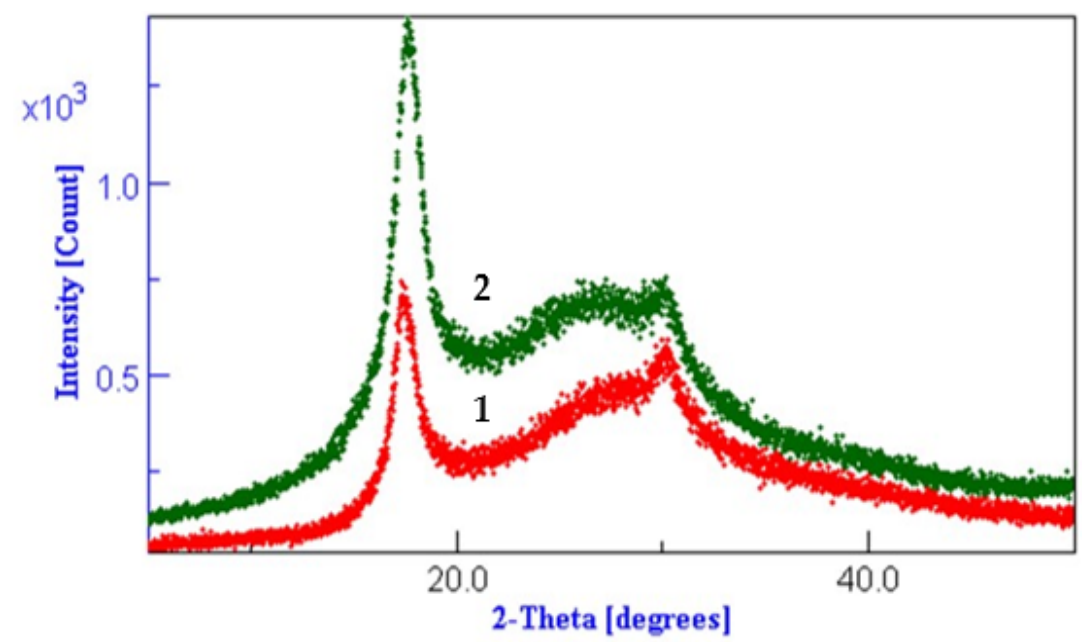

Figure 6. Diffractograms of Melana treated at $120{ }^{\circ} \mathrm{C}$ for $30 \mathrm{~min}$ with excess of reagents: (1) control sample, and (2) sample V1 with $\mathrm{KOH}$ plus iodine.

\subsection{SEM Plus Map Plus EDX Results}

Results of quantitative and qualitative analyses provided by EDX and SEM will be discussed below. The results of the EDX quantitative elemental analysis of untreated and functionalized Melana are presented in Table 4 and Figure 7. The weight percent (wt.\%), weight percentage (norm. wt.\%) and atomic percentage (norm. at.\%) of the elements present in the sample were calculated.

Table 4. Elemental composition of Melana fibers before and after functionalization.

\begin{tabular}{cccccccc}
\hline \multicolumn{4}{c}{ Untreated Melana } & \multicolumn{5}{c}{ Functionalized Melana } \\
\hline Element & [wt.\%] & [norm. wt.\%] & [norm. at. $\%$ ] & Element & [wt.\%] & [norm. wt. $\%$ ] & [norm. at. $\%$ ] \\
\hline Carbon & 51.47189 & 51.47343 & 56.24074 & Carbon & 45.25431 & 45.25567 & 50.63058 \\
\hline Nitrogen & 34.86316 & 34.86421 & 32.66563 & Nitrogen & 37.58808 & 37.58921 & 36.06172 \\
\hline Sulfur & 0.274572 & 0.27458 & 0.112376 & Sulfur & 0.421683 & 0.421696 & 0.176715 \\
\hline Oxygen & 13.38738 & 13.38778 & 10.98125 & Oxygen & 15.47541 & 15.47587 & 12.99782 \\
\hline & & & & Iodine & 1.257518 & 1.257556 & 0.133159 \\
\hline Sum & 99.997 & 100 & 100 & Sum & 99.997 & 100 & 100 \\
\hline
\end{tabular}




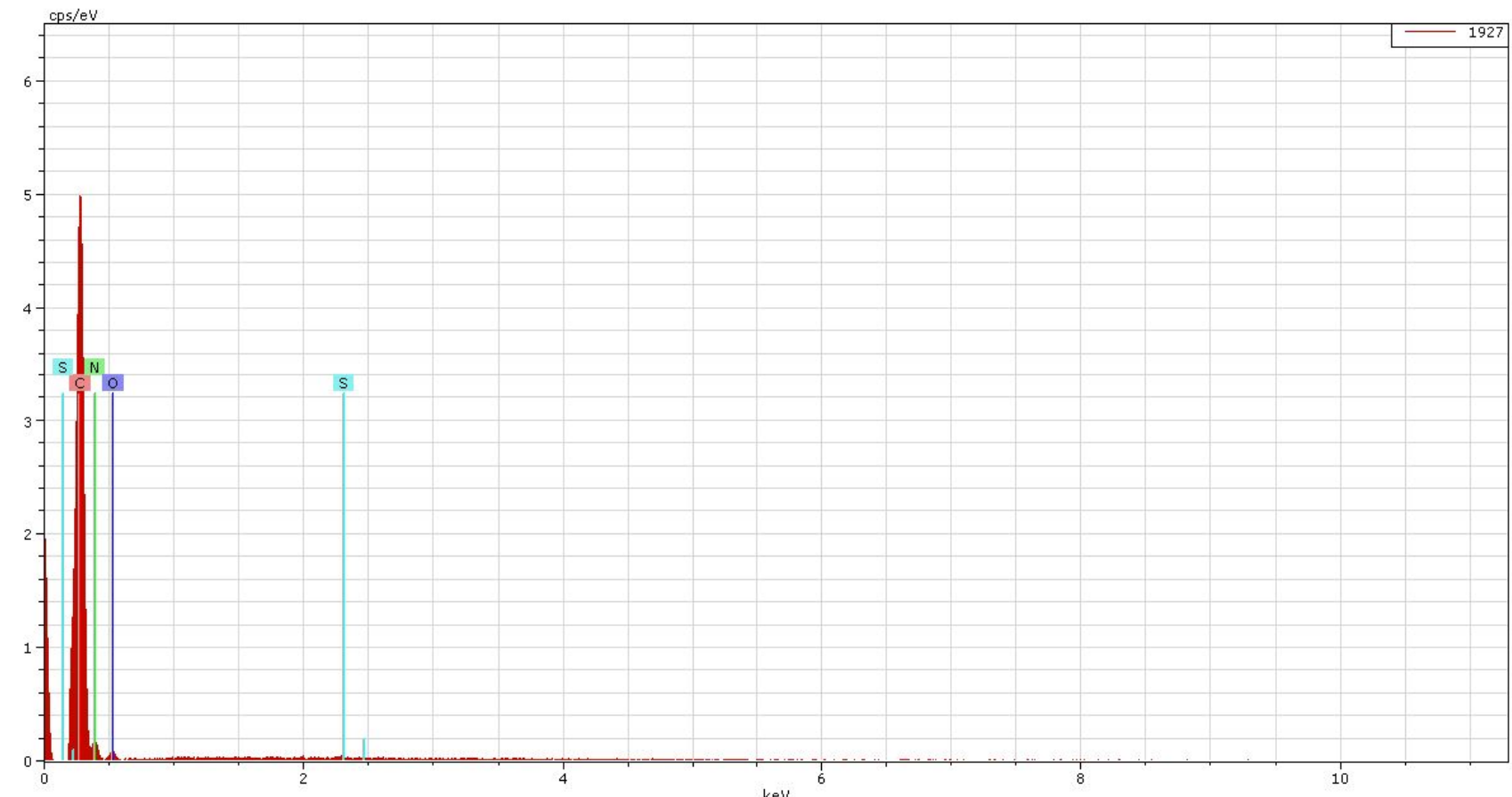

(a)

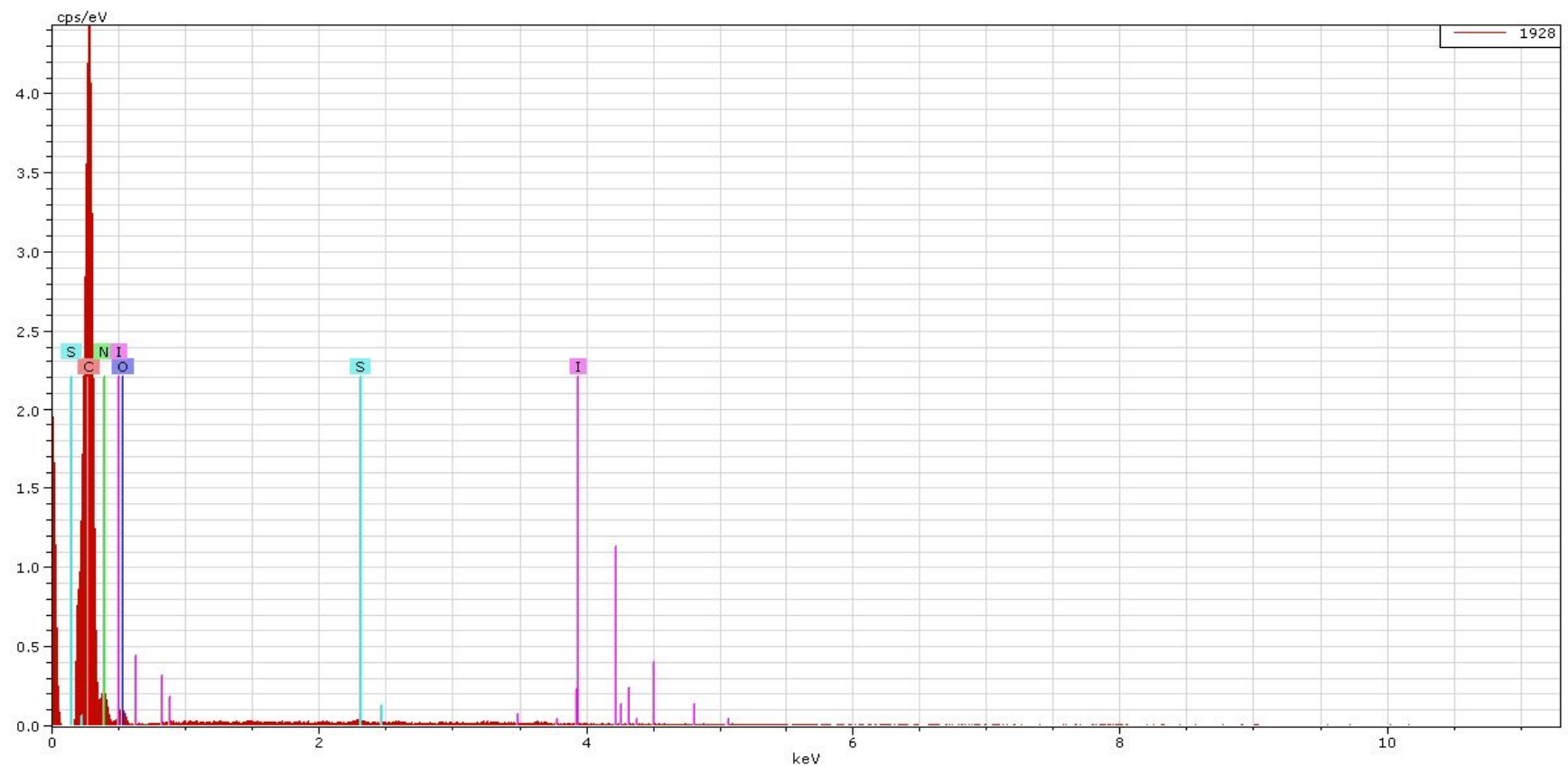

(b)

Figure 7. EDX spectrum of element analysis for Melana fibers: (a) untreated, and (b) after functionalization.

Quantitative analysis indicates the increase of oxygen $\left(\mathrm{O}_{2}\right)$ content, which was due to generation of new $-\mathrm{COOH}$ functional groups. Carbon content decreased as a result of the ester group of VA splitting and the elimination of acetic acid, $\mathrm{CH}_{3} \mathrm{COOH}$. The presence of iodine in functionalized Melana (Table 4) proved the formation of the iodine-oxime group $(\mathrm{I}-\mathrm{C}=\mathrm{N}-\mathrm{OH})$ and the persistence of iodine in the acrylic polymer, even after postfunctionalization washing of samples. The emergence of $\mathrm{C}=\mathrm{N}$ groups is associated with color change from white to shades of yellow and brown, as stated in literature [14,42].

Qualitative elemental analysis of Melana indicated the presence of $C, O, N, S$ atoms and the apparition of iodine in functionalized fibers, in addition to the above-mentioned elements (Figure 7). 
The presence, in Melana, of elements identified by EDX analysis (Table 4 and Figure 7) was confirmed by SEM plus map/microphotography results. Element maps recorded on Melana fibers (Figures 8 and 9) showed the presence of C, N, O and S atoms. Sulphur as found in the terminal $\mathrm{SO}_{3} \mathrm{H}$ groups of the acrylic polymer.

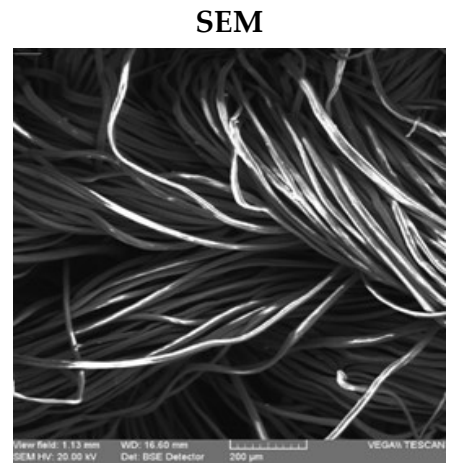

Nitrogen

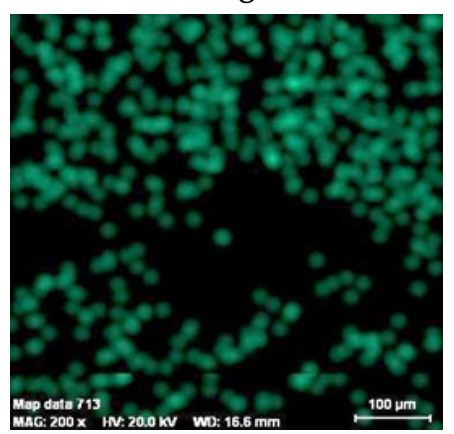

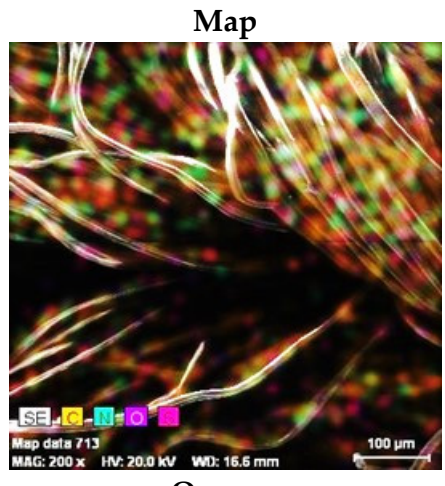

Oxygen

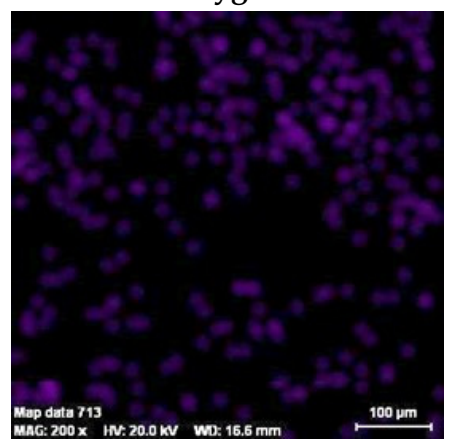

Carbon

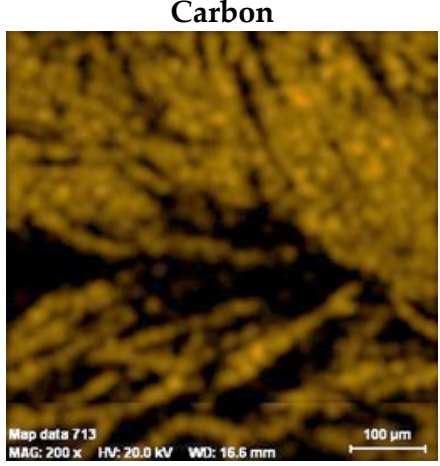

Sulfur

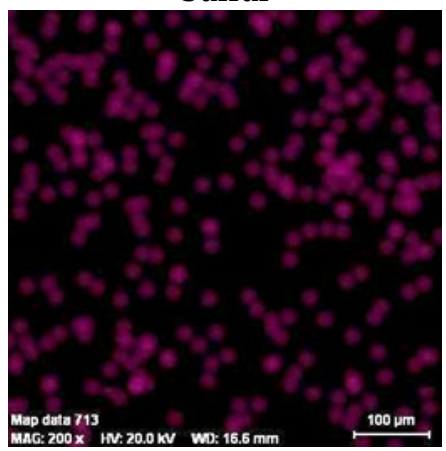

Figure 8. SEM image and element mapping in untreated Melana fibers.

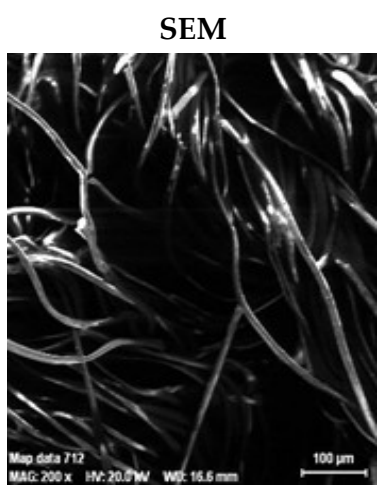

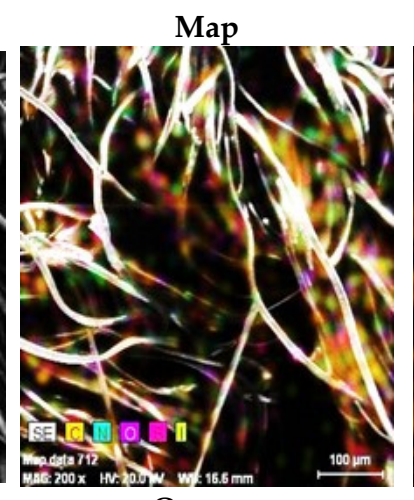

Oxygen
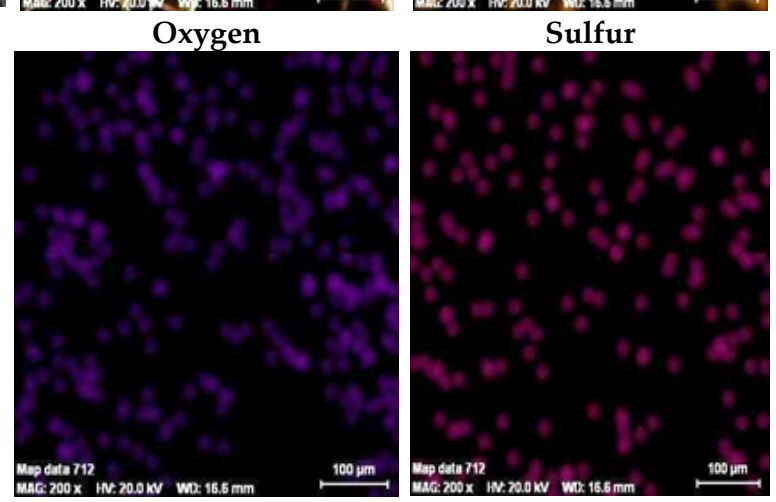

Carbon

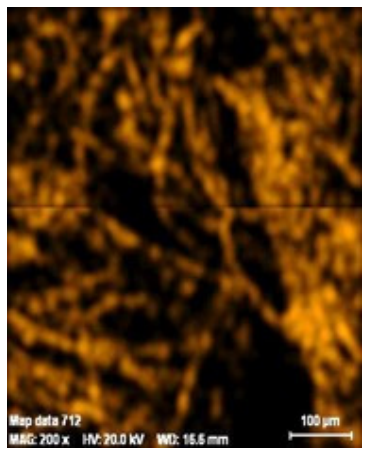

Sulfur

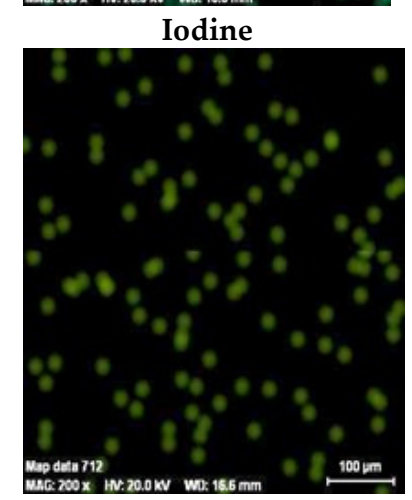

Figure 9. SEM image and element mapping in functionalized Melana fibers. 
The images qualitatively confirm the EDX results presented previously, namely the presence of iodine in a percentage of $1.257518 \%$ and an increase in oxygen content in functionalized Melana (15.47541\% compared to $13.38738 \%$ in untreated Melana).

The element map of iodine confirmed the emergence of iodine-oxime groups during functionalization.

The results of the EDX analysis for the Dralon L fibers are given in Table 5 and Figure 10.

Table 5. Elemental composition of Dralon L fibers before and after functionalization.

\begin{tabular}{|c|c|c|c|c|c|c|c|}
\hline \multicolumn{3}{|c|}{ Untreated Dralon L } & \multirow[b]{2}{*}{ [norm.at.\%] } & \multirow[b]{2}{*}{ Element } & \multicolumn{2}{|c|}{ Functionalized Dralon L } & \multirow[b]{2}{*}{ [norm.at.\%] } \\
\hline Element & {$[w t . \%]$} & [norm.wt.\%] & & & [wt.\%] & [norm.wt.\%] & \\
\hline Carbon & 51.66247 & 51.66402 & 56.31075 & Carbon & 43.36094 & 43.36224 & 49.15906 \\
\hline Nitrogen & 36.4963 & 36.49438 & 34.10928 & Nitrogen & 37.1662 & 37.16731 & 36.13237 \\
\hline Sulfur & 0.266486 & 0.266494 & 0.108799 & Sulfur & 0.388544 & 0.388556 & 0.164998 \\
\hline \multirow[t]{2}{*}{ Oxygen } & 11.57476 & 11.57511 & 9.471172 & Oxygen & 16.80041 & 16.80091 & 14.29883 \\
\hline & & & & Iodine & 2.280914 & 2.280983 & 0.244746 \\
\hline Sum & 100 & 100 & 100 & Sum & 100 & 100 & 100 \\
\hline
\end{tabular}

The Quantitative elemental analysis showed that the oxygen content of untreated Dralon L (11.57\%) was lower than that of untreated Melana (13.38\%).

For Dralon L, results of EDX analysis show that:

1. Functionalization increased the oxygen content of the polymer, from $11.57 \%$ in the treated sample, to $16.80 \%$ in the functionalized sample;

2. Compared to the untreated polymer, presence of iodine was noticed (in concentration of $2.28 \%$; ; and

3. The degree of functionalization can be associated with the iodine content $(2.28 \%)$ of the treated fiber.

Elements present in Dralon L's chemical composition are visualized in the corresponding element maps from Figures 11 and 12.

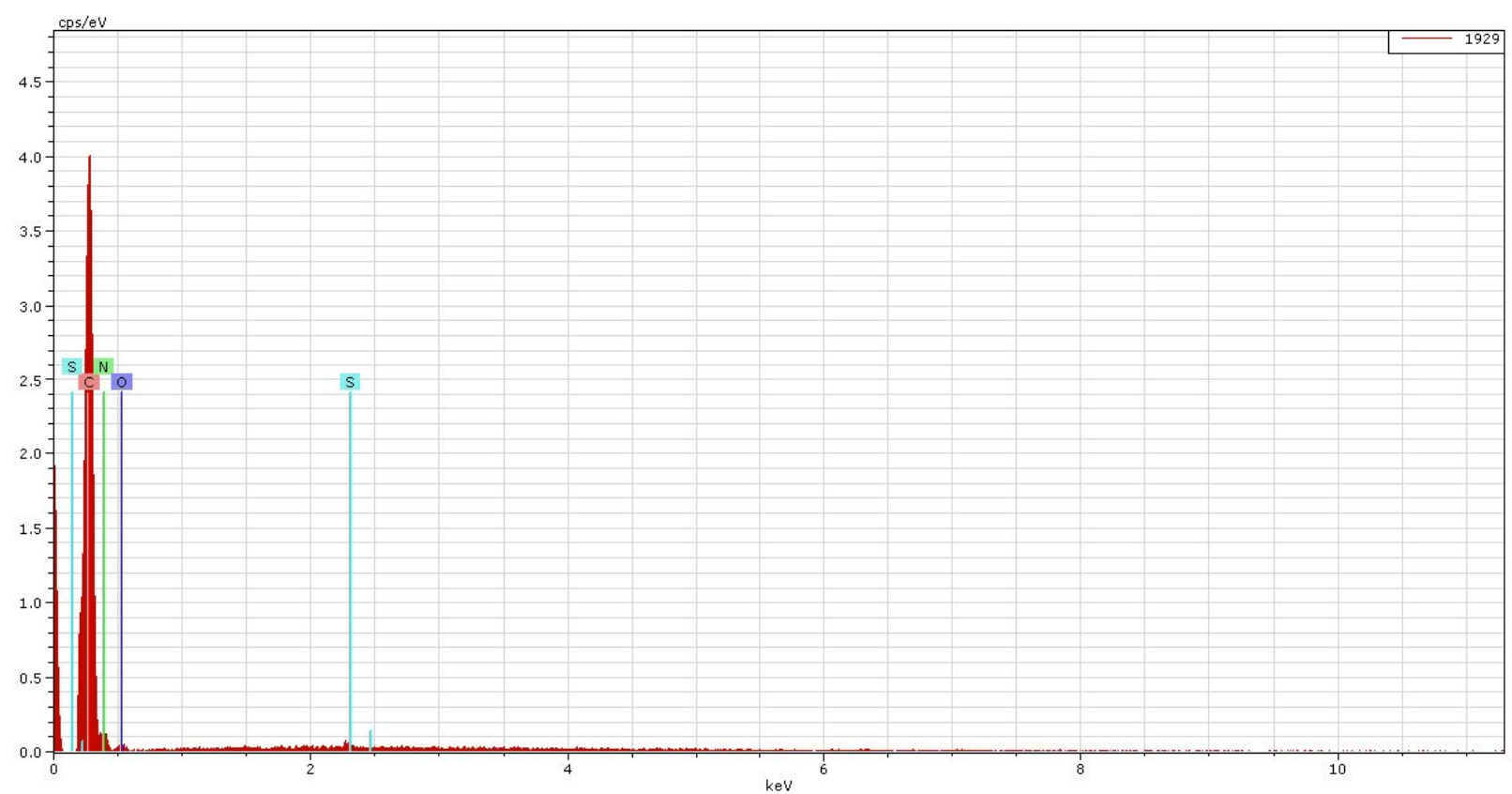

(a)

Figure 10. Cont. 


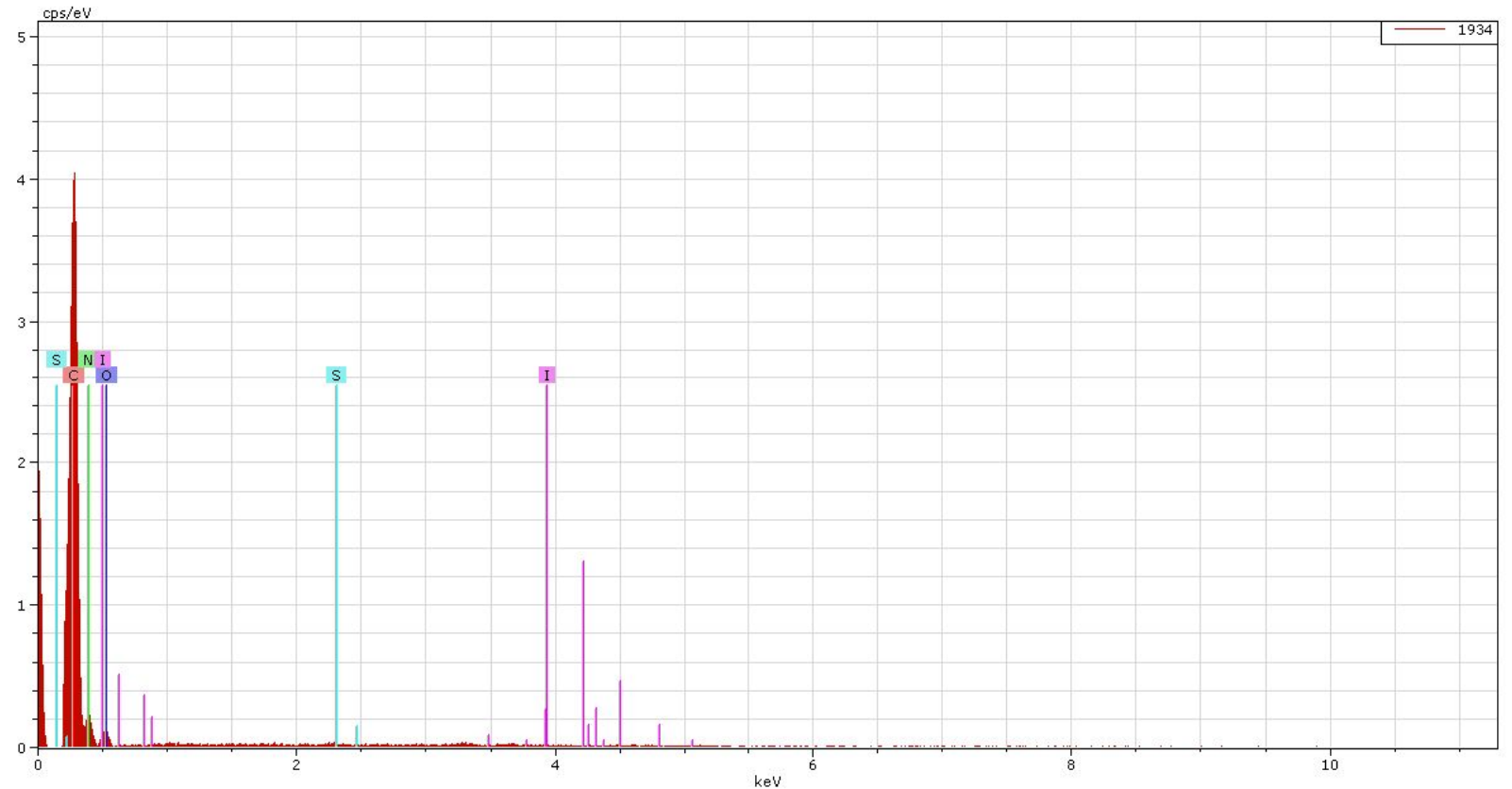

(b)

Figure 10. EDX spectrum of element analysis for Dralon L fibers: (a) untreated, and (b) after functionalization.
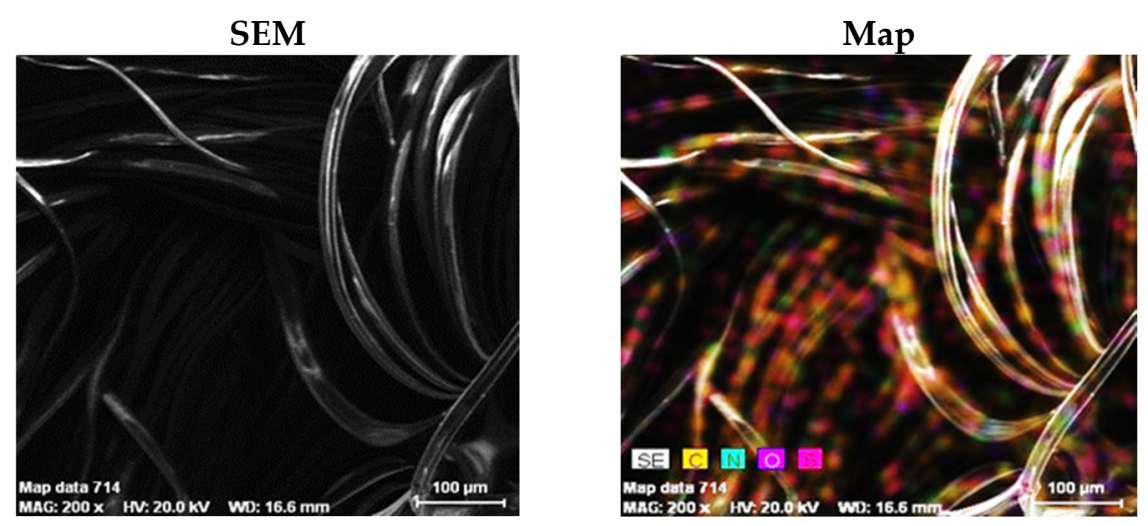

Oxygen
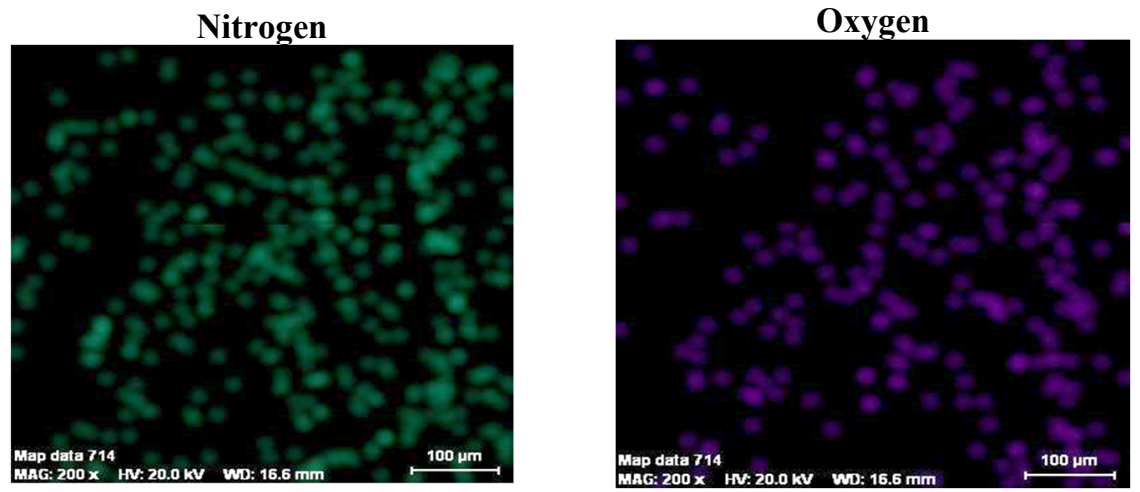

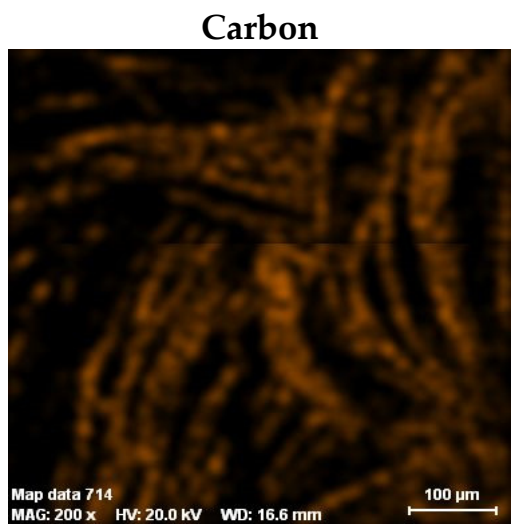

Sulfur

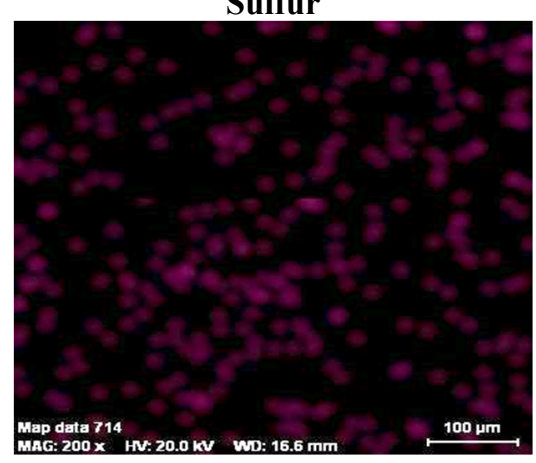

Figure 11. SEM image and elements mapping of untreated Dralon L fibers. 


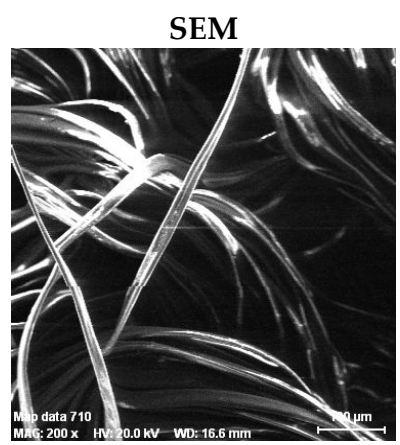

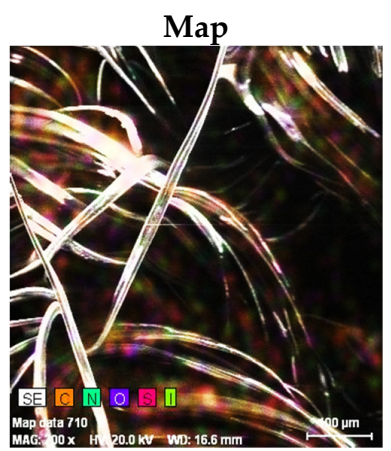

Oxygen

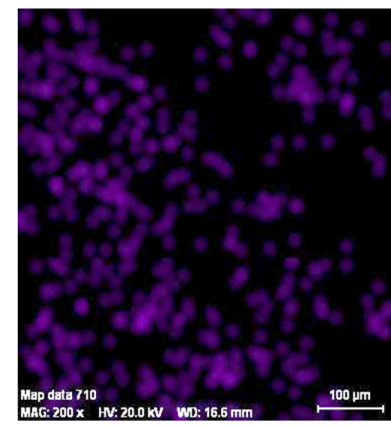

Carbon

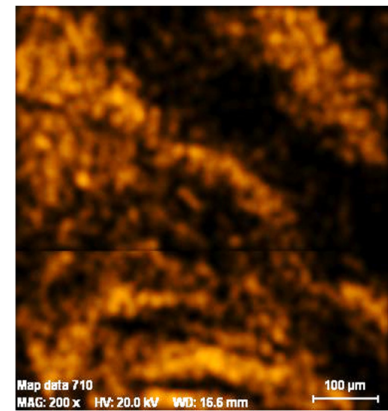

Sulfur

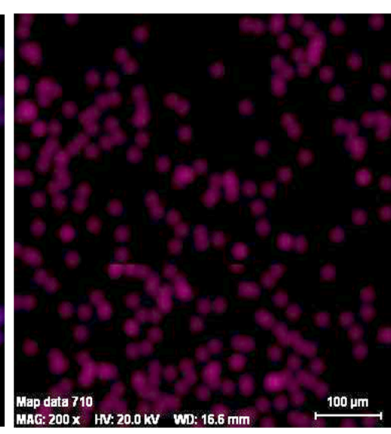

Nitrogen

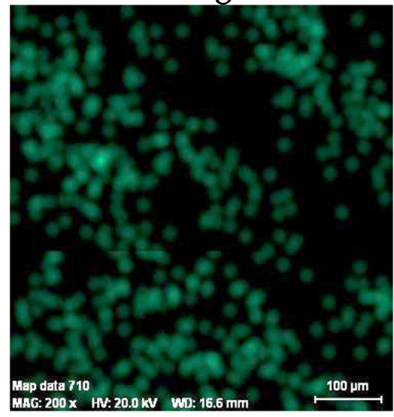

Iodine

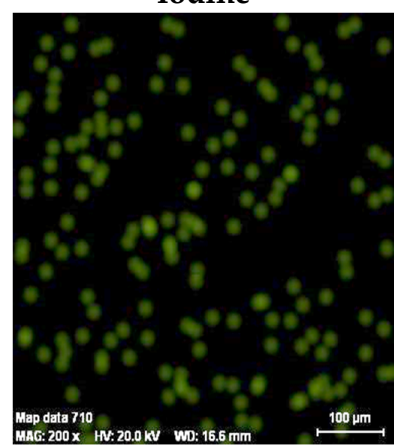

Figure 12. SEM image and elements mapping of functionalized Dralon L fibers.

A correlation between EDX results and SEM images of Dralon L allows the following observations:

(a) The iodine content of functionalized Dralon L $(2.28 \%)$ is higher than that of functionalized Melana (1.25\%);

(b) The oxygen content of functionalized Dralon L (16.80\%) is higher than that of pristine Dralon L fiber (11.57\%); and

(c) The functionalization degree of Dralon $\mathrm{L}$ is equal to $2.28 \%$ and identical to its iodine content.

\subsection{Thermal Resistance of Functionalized Fibers}

The values of thermal resistance before and after functionalization of PAN fibers with $\mathrm{KOH}+\mathrm{I}_{2} / \mathrm{KI}$ are given in Figure 13, together with the results of statistical analysis.

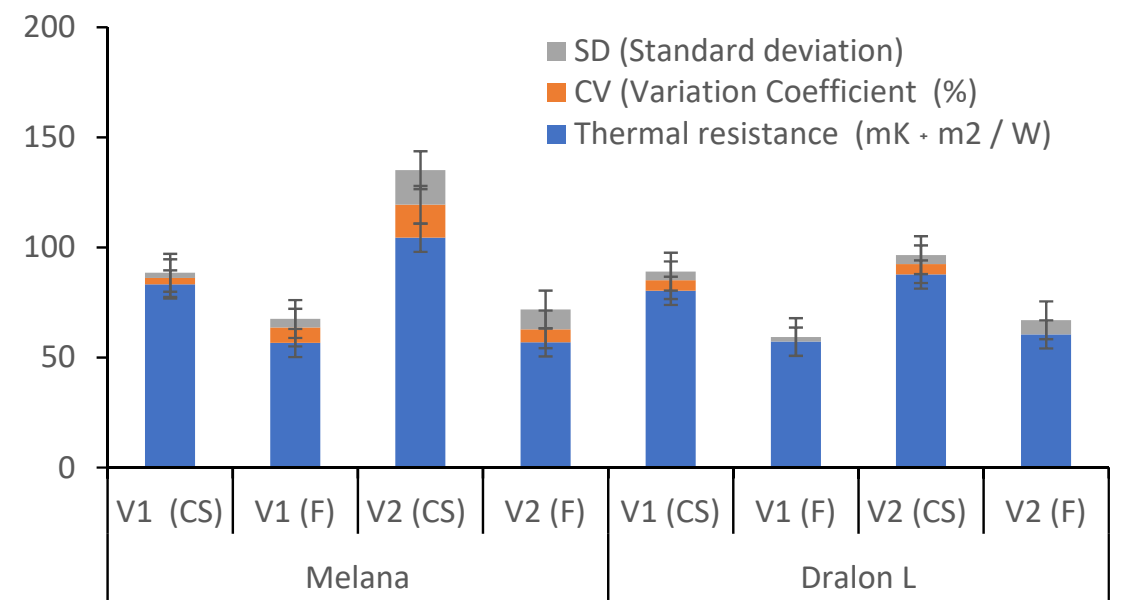

Figure 13. Influence of the functionalization treatment on the thermal resistance of two knitted fabric variants (V1 and V2) with acrylic fibers Melana and Dralon L. (abbreviation: CS = control sample; $\mathrm{F}=$ functionalization). 
In Figure 13, means of 5 determination per sample are figured, together with the results of the statistical analysis. Functionalization treatments determine the decrease of thermal resistance and increase of thermal conductivity, consequently.

Due to the specific texture of knitted fabric resulting from the curly stitch configuration, gauge value directly influenced fabric thermal resistance.

\subsection{Results of Thermogravimetric Analysis}

Thermogravimetric curves of untreated and functionalized PAN fibers (Figure 14) gave information regarding thermal and oxidative stability of the studied polymers.

As shown in Figure 14, both the untreated and functionalized samples underwent one-step mass changes. Thermal stability was related to the onset temperature $\left(\mathrm{T}_{\text {onset }}\right)$, i.e., the higher to $\mathrm{T}_{\text {onset }}$ value, the higher thermal stability. The increase of the thermal stability of studied acrylic polymers due to functionalization is proven by the experimental values of $\mathrm{T}_{\text {onset: }}$ :

- $\mathrm{T}_{\text {onset }}$ of untreated Melana $=640{ }^{\circ} \mathrm{C}$;

- $\mathrm{T}_{\text {onset }}$ of functionalized Melana $=680{ }^{\circ} \mathrm{C}$;

- $\mathrm{T}_{\text {onset }}$ of untreated Dralon $\mathrm{L}=670{ }^{\circ} \mathrm{C}$; and

- $\mathrm{T}_{\text {onset }}$ of functionalized Dralon $\mathrm{L}=690^{\circ} \mathrm{C}$.

This behavior can be related to the presence of iodine in the functionalized fibers.

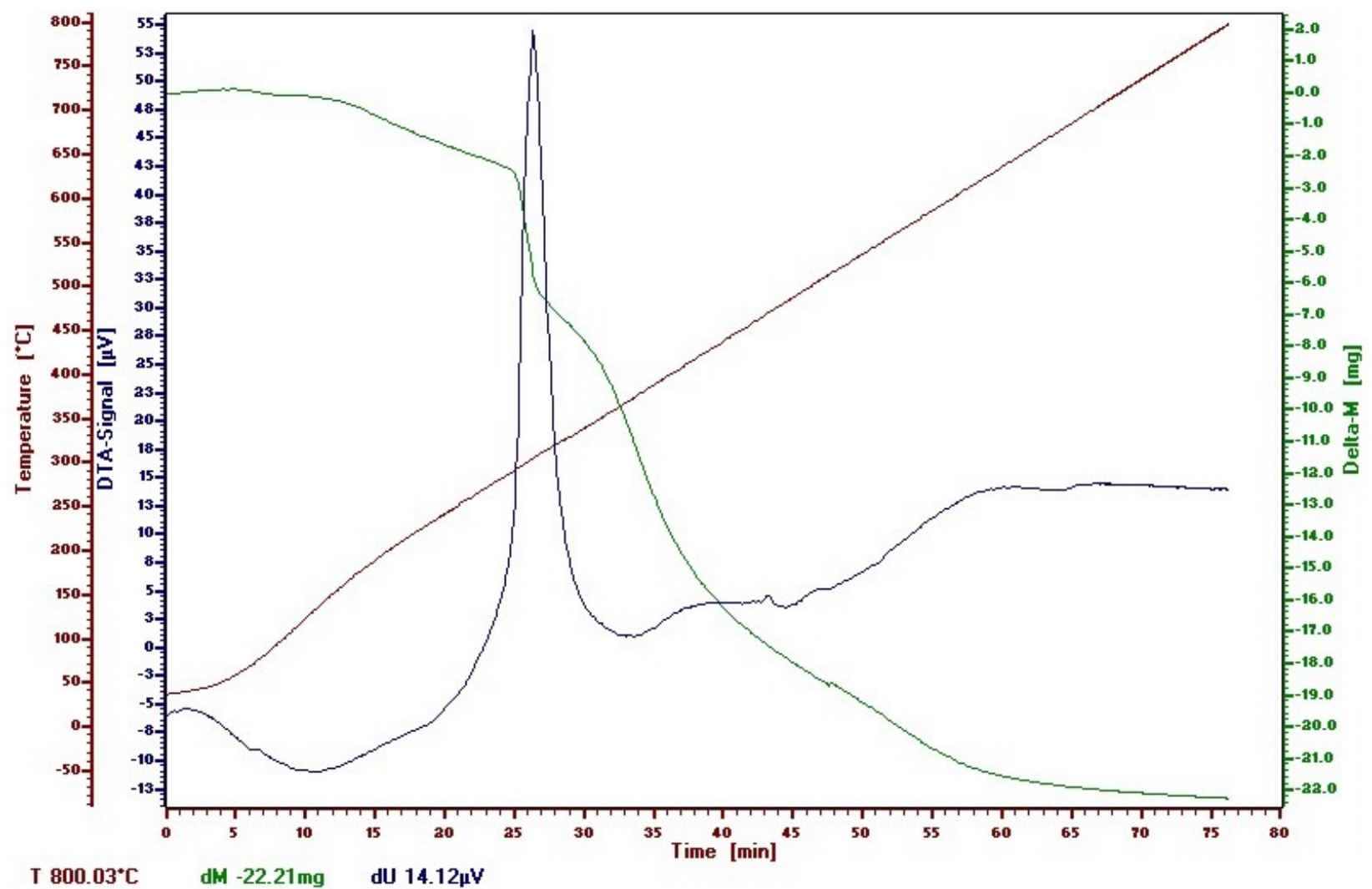

(a)

Figure 14. Cont. 


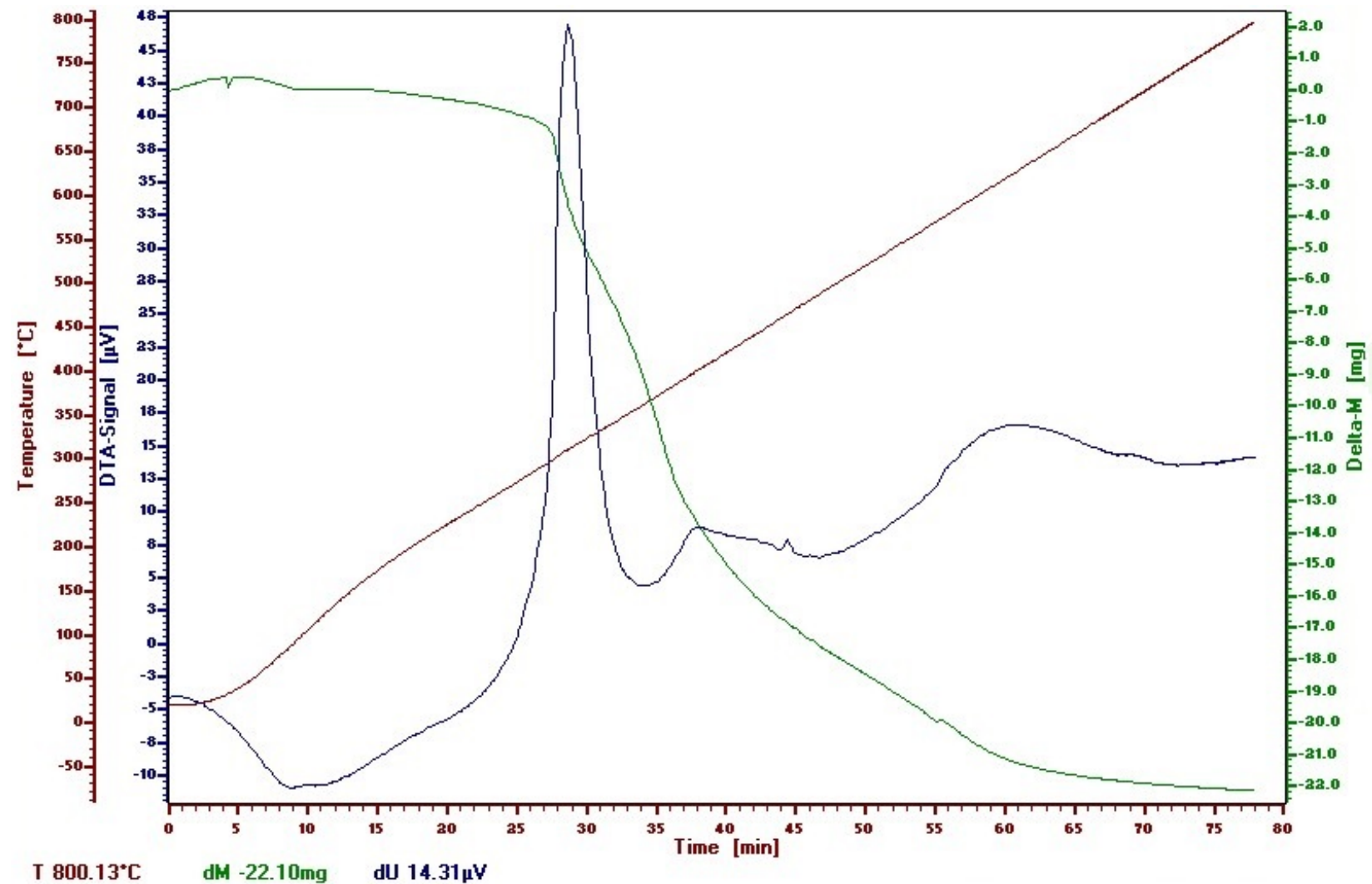

(b)

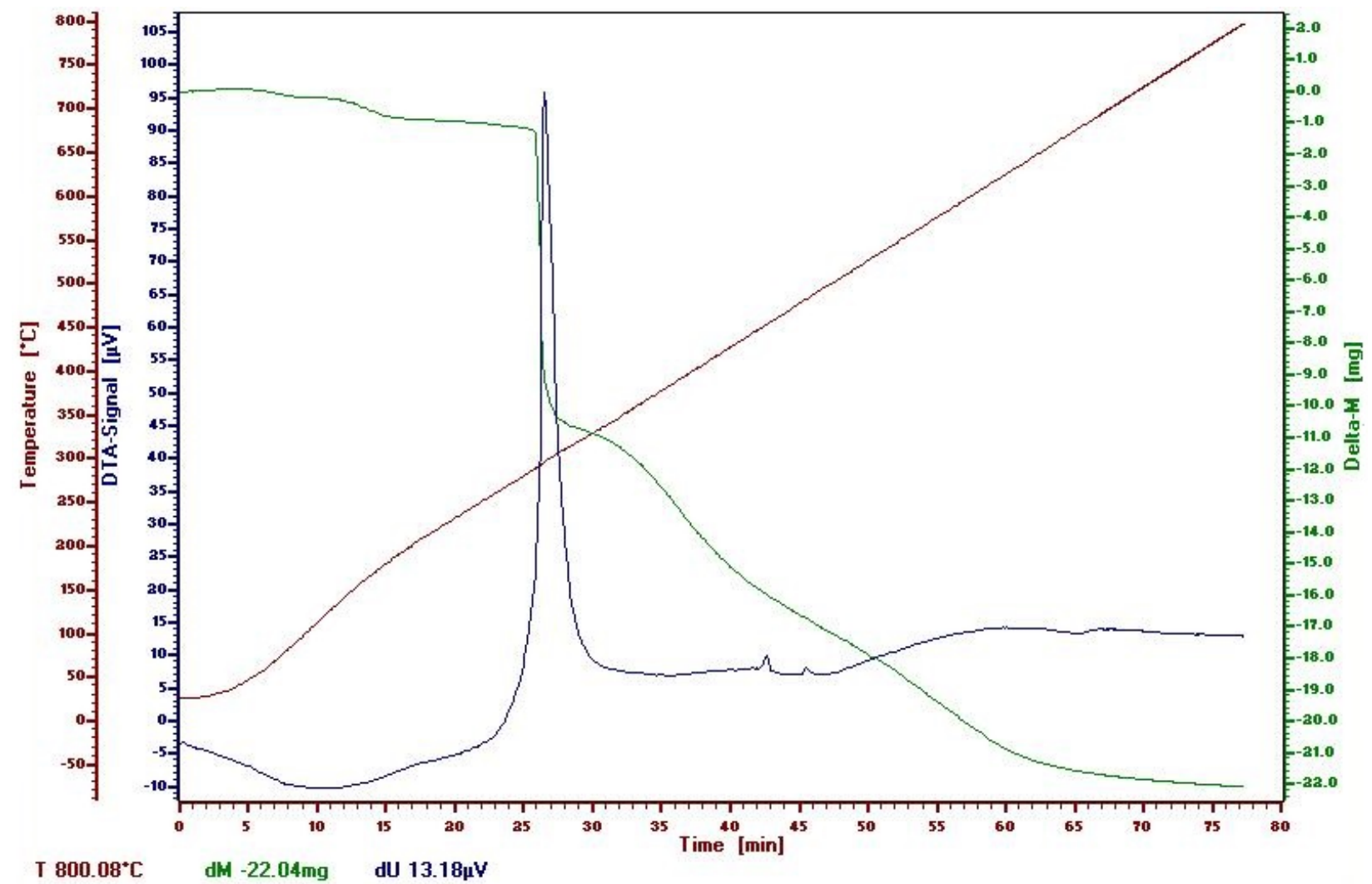

(c)

Figure 14. Cont. 


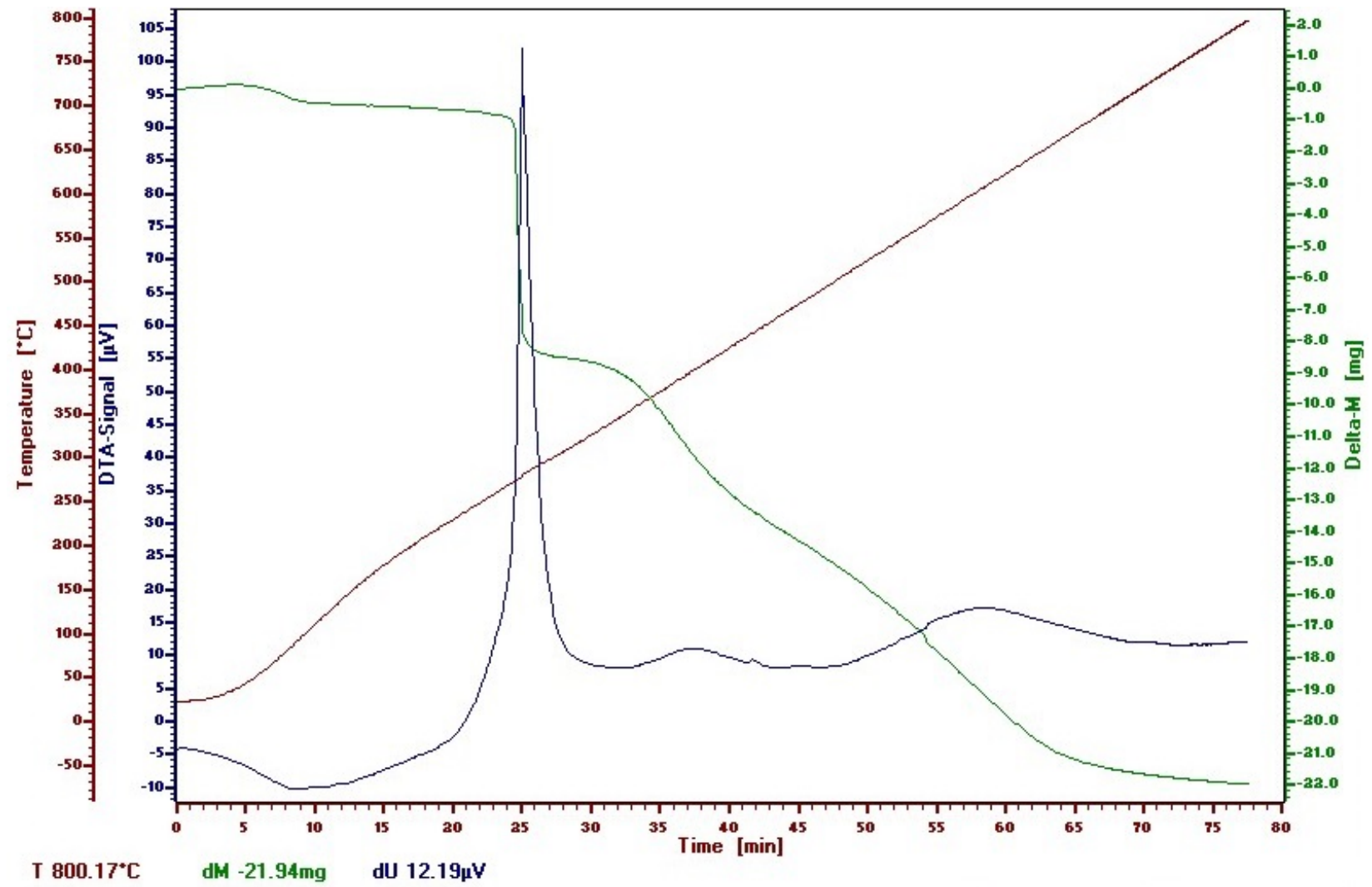

(d)

Figure 14. Experimental TG and DTA curves for acrylic polymers: control sample Melana (a), functionalized Melana (b), control sample Dralon L (c), functionalized Dralon L (d).

\subsection{Colorimetric Measurements}

3.7.1. Colorimetric Measurements on Samples Subjected to Severe Functionalization

Tinting of functionalized PAN was assessed by colorimetric measurements, i.e., color location on the yellow-blue axis $\left(b^{*}\right)$ and color strength (K/S) (Figure 15).

Severe functionalization treatments (with excess of chemicals, $\mathrm{I}_{2} / \mathrm{KI}+\mathrm{KOH}$ ) resulted in tinting degrees higher than standard values. Optimal treatment (controlled functionalization) parameters must be established to protect the fabric and maximize the $b$ * and K/S values at the same time.

\subsubsection{Colorimetric Measurements on Samples Subjected to Controlled Functionalization}

Tinting in yellow-orange shades of acrylic fabrics is obvious even with low concentrations of functionalization agents (Table 6), which is proven by:

- The increase of $b$ * values, as compared with the standard;

- Decrease of luminosity, $\mathrm{L}^{*}$; and

- Increase of saturation, $C^{*}$. 

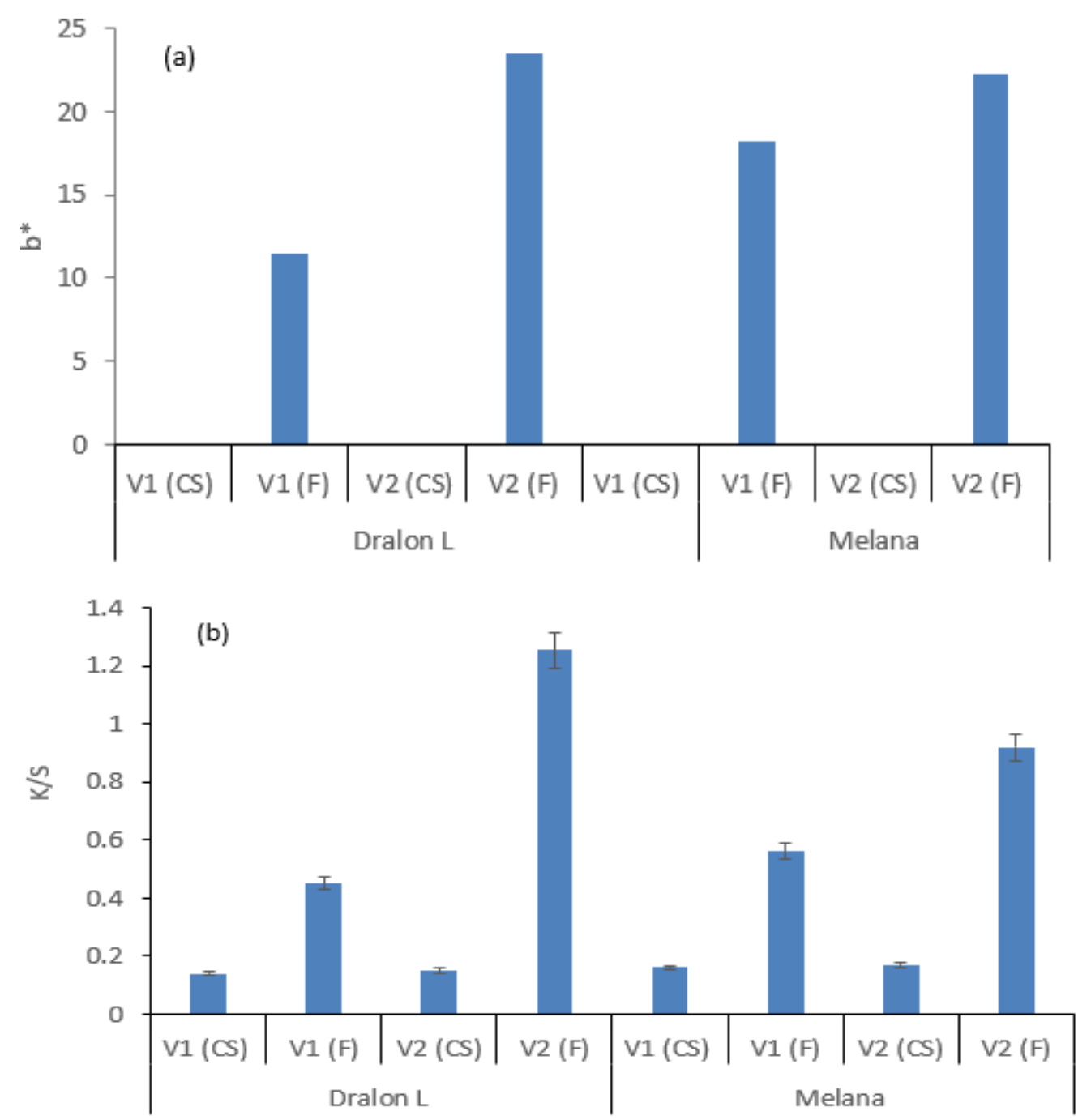

Figure 15. Colorimetric measurements on control sample (CS) and functionalized (F) Melana and Dralon L: (a) b * values; (b) K/S, color intensity.

\subsection{Influence of Functionalization Treatment upon the Color Intensity (K/S)}

The influence of functionalization treatment upon K/S value is displayed in Figure 16. Increase of color intensity $(\mathrm{K} / \mathrm{S})$ is more noticeable in the case of controlled functionalization than in the case of severe functionalization, when chemical reagents were used in excess:

1. Color intensity depends on the functionalization degree; and

2. Color intensity depends on the working parameters and the functionalization agents:

- Treatment with $\mathrm{I}_{2} / \mathrm{KI}+\mathrm{KOH}$ at room temperature did not produce any color change of the acrylic fibers: $\mathrm{b}^{*}$ values increased from 0.01 for untreated Dralon $\mathrm{L}$ to 1.08 for Dralon L functionalized with $9 \% \mathrm{KOH}+9 \% \mathrm{I}_{2} / \mathrm{KI}$.

- Functionalization at $120{ }^{\circ} \mathrm{C}$ for 60 min determined a noticeable increase of $b^{*}$ value: 17.67 after Dralon L treatment with $3 \% \mathrm{KOH}+3 \% \mathrm{I}_{2} / \mathrm{KI}$ and 20.7-28.47 after treatment with the same reagents but in concentrations of $6-9 \%$.

- Functionalization resulted in fiber coloration in yellow-orange shades without using dyes. 
Table 6. CIELab values of acrylic fibers after controlled functionalization.

\begin{tabular}{|c|c|c|c|c|c|c|}
\hline Sample Name & $L^{*}$ & $a^{*}$ & $\mathbf{b}^{*}$ & $C^{*}$ & h & $\mathrm{K} / \mathrm{S}$ \\
\hline \multicolumn{7}{|c|}{ Dralon $\mathrm{L}$ treatment for 3 days, at room temperature } \\
\hline Control sample & 88.72 & -0.74 & 0.01 & 0.74 & 179.42 & 0.1453 \\
\hline $9 \% \mathrm{KOH}$ & 87.47 & -1.22 & 2.48 & 2.75 & 116.13 & 0.1472 \\
\hline $9 \% \mathrm{I}_{2} / \mathrm{KI}$ & 87.98 & -1.66 & 3.57 & 3.94 & 114.95 & 0.1923 \\
\hline $9 \% \mathrm{KOH}+9 \% \mathrm{I}_{2} / \mathrm{KI}$ & 86.26 & -0.86 & 1.08 & 1.38 & 128.73 & 0.136 \\
\hline \multicolumn{7}{|c|}{ Treatment with $3 \% \mathrm{KOH}+3 \% \mathrm{I}_{2} / \mathrm{KI}$ at $120^{\circ} \mathrm{C}, \mathrm{t}=60 \mathrm{~min}, \mathrm{M}=1: 15$} \\
\hline Melana & 86.84 & -0.99 & 13.16 & 13.20 & 94.29 & 0.315 \\
\hline Dralon L & 82.29 & -2.45 & 17.67 & 17.84 & 97.91 & 0.908 \\
\hline \multicolumn{7}{|c|}{ Dralon $L$ functionalization with $6 \%$ reagents at $120^{\circ} \mathrm{C}, \mathrm{t}=60 \mathrm{~min}, \mathrm{M}=1: 15$} \\
\hline $\begin{array}{c}\text { Control sample boiled at } 120^{\circ} \mathrm{C} \\
\text { (without chemicals) }\end{array}$ & 88.6 & -1.21 & 3.48 & 3.66 & 109.23 & 0.1351 \\
\hline $6 \% \mathrm{KOH}$ & 80.66 & 1.26 & 33.29 & 33.32 & 87.86 & 2.06 \\
\hline $6 \% \mathrm{I}_{2} / \mathrm{KI}$ & 86.8 & -1.08 & 3.16 & 3.34 & 108.87 & 0.1622 \\
\hline $6 \% \mathrm{KOH}+6 \% \mathrm{I}_{2} / \mathrm{KI}$ & 86.92 & -1.56 & 20.73 & 20.79 & 94.31 & 1.676 \\
\hline \multicolumn{7}{|c|}{ Dralon $L$ functionalization with $9 \%$ reagents at $120^{\circ} \mathrm{C}, \mathrm{t}=60 \mathrm{~min}, \mathrm{M}=1: 15$} \\
\hline $\begin{array}{c}\text { Control sample boiled at } 120{ }^{\circ} \mathrm{C} \\
\text { (without chemicals) }\end{array}$ & 88.6 & -1.21 & 3.48 & 3.66 & 109.23 & 0.1351 \\
\hline $9 \% \mathrm{KOH}$ & 82.56 & -0.71 & 24.85 & 24.86 & 91.63 & 1.171 \\
\hline $9 \% \mathrm{I}_{2} / \mathrm{KI}$ & 87.02 & -1.12 & 3.51 & 3.68 & 107.72 & 0.170 \\
\hline $9 \% \mathrm{KOH}+9 \% \mathrm{I}_{2} / \mathrm{KI}$ & 81.43 & -0.02 & 28.47 & 28.47 & 90.03 & 1.530 \\
\hline
\end{tabular}

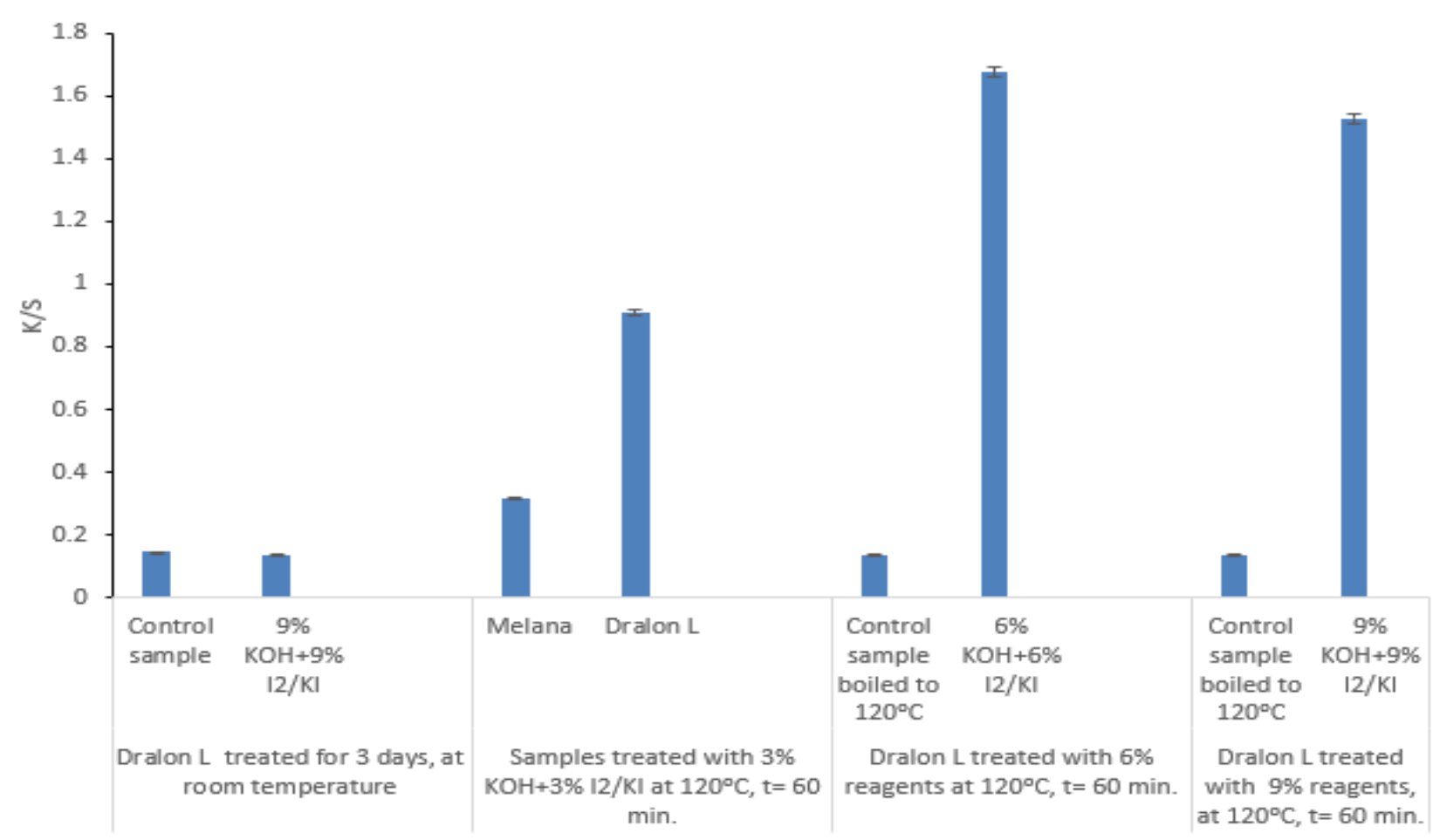

Figure 16. Dependence of color intensity on the functionalization treatment.

The generation of new functional groups of iodine-oxime types $(\mathrm{I}-\mathrm{C}=\mathrm{N}-\mathrm{OH})$ by the conversion of a part of fraction of nitrile groups $(-\mathrm{C} \equiv \mathrm{N})$ determines the increase of the tinctorial capacity of acrylic fibers and creates the possibility of dyeing with acid dyes, in acid media, which is not typical for PAN fibers. The emergence of the iodine-oxime functional group was confirmed by the starch test [31,32], when the specific blue-black color that indicates the presence of $\mathrm{I}_{3}{ }^{-}$in a free state (if doping takes place) did not appear. 
Data from Figure 16 show that functionalization led to the generation of iodine-oxime groups and the coloration of the acrylic fibers in yellow-orange (proved by the K/S values in range 0.15 to 1.676 ).

Functionalized fibers can be dyed in uniform and deep colors with non-typical dyes, such as the acid dyes C.I. Acid Red 57 (Figure 17c,d) and C.I. Acid Violet 48 (Figure 17c', $\mathrm{d}^{\prime}$ ).

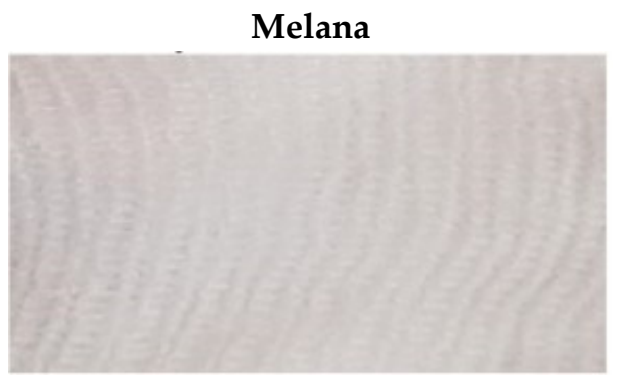

(a)

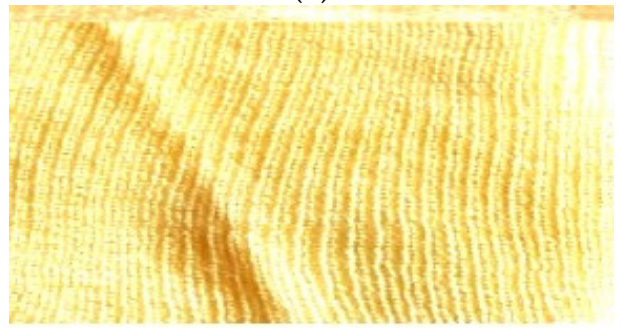

(b)

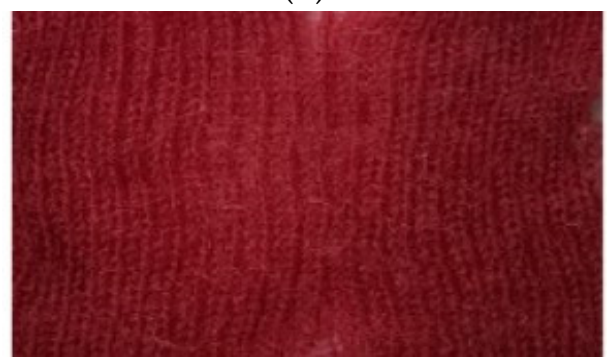

(c)

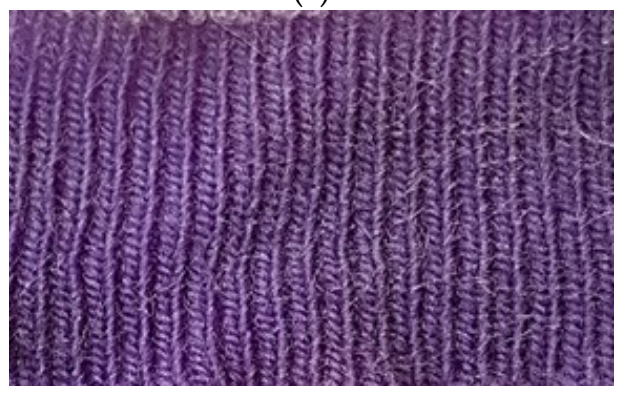

(d)
Dralon L

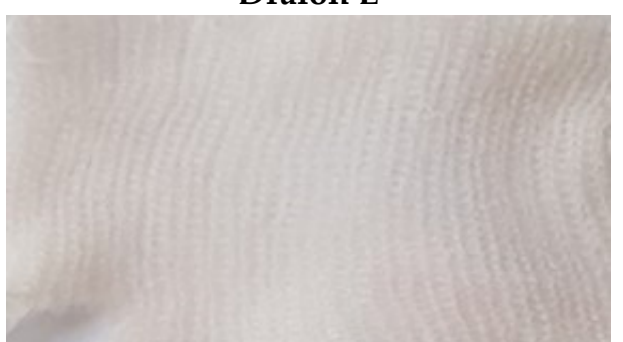

$\left(\mathbf{a}^{\prime}\right)$

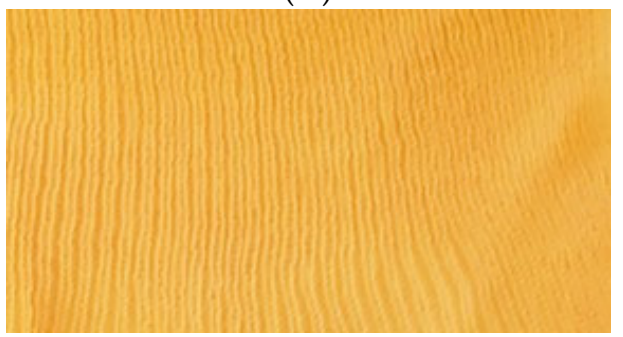

$\left(\mathbf{b}^{\prime}\right)$

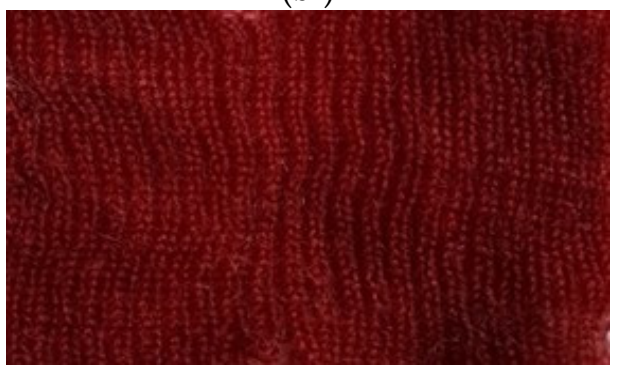

$\left(\mathbf{c}^{\prime}\right)$

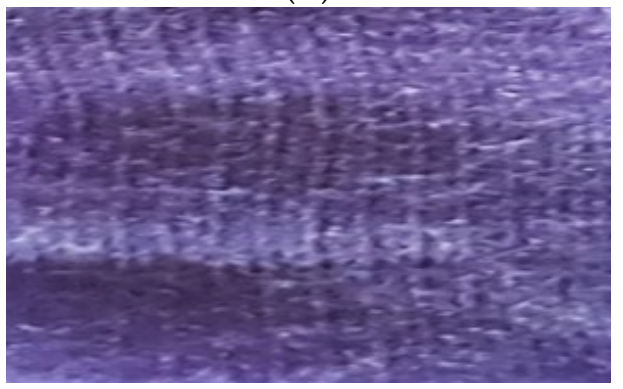

$\left(d^{\prime}\right)$

Figure 17. Color of acrylic fibers in different treatment stages: $\left(\mathbf{a}, \mathbf{a}^{\prime}\right)$ untreated, $\left(\mathbf{b}, \mathbf{b}^{\prime}\right)$ functionalized, and $\left(\mathbf{c}-\mathbf{d}^{\prime}\right)$ functionalized and dyed with acid dyes (C.I. Acid Red 57 and C.I. Acid Violet 48).

The functionalization of Melana and Dralon L fibers was confirmed by:

1. Coloration in yellow-orange, with shades depending on the treatment severity ( $b$ and $\mathrm{b}^{\prime}$ images in Figure 17); and

2. Affinity for non-specific/non-typical dyes and good color fastness (c, $\mathrm{c}^{\prime}$ and $\mathrm{d}, \mathrm{d}^{\prime}$ images in Figure 17). 


\section{Conclusions}

The results of this study highlight the effects of $\mathrm{KIO}_{3}$ (obtained in situ) on pre-oxidized and colored PAN fibers, and may open a new way to obtain the coloration of the polymer during functionalization.

Functionalization experiments on acrylic fibers Melana and Dralon L revealed:

- Coloration of PAN fibers could be a result of functionalization only, without the need to use dyestuffs;

- Coloration of treated PAN fibers depended on the functionalization degree;

- Functionalization was confirmed by the results of FTIR, SEM, Map and thermogravimetric analyses;

- Functionalization improved the fabric wearing comfort, due to the increase of fiber thermal conductibility;

- Functionalization resulted in chemical modifications of the copolymer chemical structure by conversion of some nitrile groups $(\mathrm{C} \equiv \mathrm{N})$ into oxime groups and the alteration of the crystalline/amorphous ratio;

- The yellow-orange color gained by functionalization exhibited fastness to repeated washings and boiling at acidic $\mathrm{pH}$; and

- Functionalization of the Melana and Dralon L fibers can be easily proven by the tinctorial method, which consists in dyeing with dyes compatible with the functional group created by functionalization.

Author Contributions: Conceptualization, V.P. and P.C.; methodology, V.P. and M.P. (Melinda Pruneanu); software, A.P.; formal analysis, V.R., P.C., B.I. and M.M.; investigation, A.D., I.N.C., F.C. and M.P. (Marius Pîslaru); writing—original draft preparation, V.P. and I.I.B.; writing-review and editing, I.I.B., M.P. (Melinda Pruneanu) and V.M.; visualization, A.C.B. and S.S.M.; supervision, I.C.; project administration, V.P. All authors have read and agreed to the published version of the manuscript.

Funding: This work was supported by a Publications grant of the Technical University "Gheorghe Asachi" of Iasi, project number P11/2021.

Institutional Review Board Statement: Not applicable.

Informed Consent Statement: Not applicable.

Acknowledgments: Thanks to Corneliu Munteanu (from “Gheorghe Asachi” Technical University, Iasi) for performing SEM analysis.

Conflicts of Interest: The authors declare no conflict of interest.

\section{References}

1. Jin, S.Y.; Kim, M.H.; Jeong, Y.G.; Yoon, Y.I.; Park, W.H. Effect of alkaline hydrolysis on cyclization reaction of PAN nanofibers. Mater. Design 2017, 124, 69-77. [CrossRef]

2. Wojcik, G.; Neagu, V.; Bunia, I. Sorption Studies of Chromium (VI) onto New Ion Exchanger with Tertiary Amine, Quaternary Ammonium, and Ketone Groups. J. Hazard. Mater. 2011, 190, 544-552. [CrossRef] [PubMed]

3. Bunia, I.; Neagu, V.; Luca, C. Chemical Transformations of Different Acrylic Crosslinked Polymers with Primary Amines and Some Applications of the Synthesized Compounds. React. Funct. Polym 2006, 66, 871-883. [CrossRef]

4. Bandak, A.; Kantouch, A.; El-Gabry, L. Hydrazine Treatments on Acrylic Fibers for New Dyeing Opportunities. Am. Dyest. Rep. 1995, 84, 34-45.

5. Marie, M.M. Dyeing Acrylic Fibers with Acid Dyes. Am. Dyest. Rep. 1993, 9, 86

6. Saeed, K.; Haider, S.; Oh, T.-J.; Park, S.-Y. Preparation of Amidoxime-Modified Polyacrylonitrile (PAN-Oxime) Nanofibers and Their Applications to Metal Ions Adsorption. J. Membr. Sci. 2008, 322, 400-405. [CrossRef]

7. Dong, Y.; Han, Z.; Liu, C.; Du, F. Preparation and Photocatalytic Performance of Fe (III)-Amidoximated PAN Fiber Complex for Oxidative Degradation of Azo Dye under Visible Light Irradiation. Sci. Total Environ. 2010, 408, 2245-2253. [CrossRef]

8. Neghlani, P.K.; Rafizadeh, M.; Taromi, F.A. Preparation of Aminated-Polyacrylonitrile Nanofiber Membranes for the Adsorption of Metal Ions: Comparison with Microfibers. J. Hazard. Mater. 2011, 186, 182-189. [CrossRef]

9. Popescu, V.; Muresan, E.I. Performances of Chitosan Grafted onto Surface of Polyacrylonitrile Functionalized through Amination Reactions. Ind. Eng. Chem. Res. 2013, 52, 13252-13263. 
10. Popescu, V.; Sandu, I.C.A.; Popescu, G. Colorimetric Evaluation of Chemical Modifications Generated by PAN Functionalization in Acid/ basic Medium and Grafting with Chitosan. Rev. Chim. (Buchar.) 2016, 67, 74-80.

11. Popescu, V.; Sandu, I.C.A.; Popescu, G. Analysis of the Behaviour of PAN Functionalized with Basic Compounds, During Dyeing Process with Acid Dyes. Rev. Chim. (Buchar.) 2015, 66, 1997-2004.

12. Popescu, V. Multifunctionalizations of Textile Materials Highlighted by unconventional Dyeings. In Frontiers of Textile Materials: Polymers, Nanomaterials, Enzymes, and Advanced Modification Techniques, 1st ed.; Shabbir, M., Ahmed, S., Sheikh, J.N., Eds.; Scrivener Publishing LLC, Wiley: Hoboken, NJ, USA, 2020; Volume 11, pp. 219-290.

13. Popescu, V.; Radu, C.D.; Manea, L.R. Effects of the changes caused by certain chemical pretreatments performed on acrylic polymers. Ind. Textila 2010, 61, 23-30.

14. Rahaman, M.S.A.; Ismail, A.F.; Mustafa, A. A review of heat treatment on polyacrylonitrile fiber. Polym. Degrad. Stabil. 2007, 92, $1421-1432$.

15. Dang, W.; Liu, J.; Wang, X.; Yan, K.; Zhang, A.; Yang, J.; Chen, L.; Liang, J. Structural transformation of polyacrylonitrile (PAN) fibers during rapid thermal pretreatment in nitrogen atmosphere. Polymers (Basel) 2020, 12, 1-12.

16. Xiao, S.; Wang, B.; Zhao, C.; Xu, L.; Chen, B. Influence of Oxygen on the Stabilization Reaction of Polyacrylonitrile Fiber. J. Appl. Polym. Sci. 2013, 127, 2332-2338.

17. Xue, Y.; Liu, J.; Lian, F.; Liang, J. Effect of the oxygen-induced modification of polyacrylonitrile fibers during thermal-oxidative stabilization on the radial microcrystalline structure of the resulting carbon fibers. Polym. Degrad. Stabil. 2013, 98, $2259-2267$.

18. Xiao, S.; Cao, W.; Wang, B.; Xu, L.; Chen, B. Mechanism and Kinetics of Oxidation During the Thermal Stabilization of Polyacrylonitrile Fibers. J. Appl. Polym. Sci. 2012, 127, 3198-3203.

19. Chen, L.; Shen, Z.; Jie, L.; Liang, J.; Wang, X. Effects of oxygen on the structural evolution of polyacrylonitrile fibers during rapid thermal treatment. RSC Adv. 2020, 10, 6356-6361.

20. Hinrichsen, G. On the origin of order-disorder in drawn polyacrylonitrile. J. Appl. Polym. Sci. 1973, 17, 3305-3321. [CrossRef]

21. Peebles, L.H.; Abhiraman, A.S., Jr.; Bhat, G.S. Method for Stabilization of PAN-Based Carbon Fibers. U.S. Patent No. H1052, 5 May 1992.

22. Jin, X.; Li, L.; Xu, R.; Liu, Q.; Ding, L.; Pan, Y.; Wang, C.; Hung, W.; Lee, K.; Wang, T. Effects of Thermal Cross-Linking on the Structure and Property of Asymmetric Membrane Prepared from the Polyacrylonitrile. Polymers (Basel) 2018, 10, 539.

23. Mathur, R.B.; Mittal, J.; Bahl, O.P.; Sandle, N.K. Characteristics of KMnO4-modified PAN fibres-its influence on the resulting carbon fibres' properties. Carbon 1994, 32, 71-77.

24. Wang, Y.; Yin, W. Chemical Modification for PAN Fibers during Heat-treatment Process, Proceeding of The Fourth International Conference on Surface and Interface Science and Engineering. Phys. Procedia 2011, 18, 202-205.

25. Zhang, C.; Li, R.; Liu, J.; Chen, G.; Guo, S.; Xu, L.; Xiao, S.; Shen, Z. Effect of KMnO4 on chemical, crystal and microscopic structure of polyacrylonitrile fibers. Ceram. Int. 2019, 4, 17669-17674.

26. Andrews, R.D.; Miyachi, K.; Doshi, R.S. Iodine swelling of polyacrylonitrile I. Effect of orientation and evidence for a three phase structure. J. Macromol. Sci. B 1974, 9, 281-299.

27. Andrews, R.D.; Yen, R.C.; Chang, P.C. Iodine swelling of polyacrylonitrile, III: Creep yield and conformational transition of swollen polymer. J. Macromol. Sci. B 1981, 19, 729-742.

28. El-Ghamaz, A.; Diab, M.A.; Zoromba, M.S.; El-Sonbati, A.Z.; El-Shahat, O. Conducting polymers. VI. Effect of doping with iodine on the dielectrical and electrical conduction properties of polyacrylonitrile. Solid State Sci. 2013, 24, 140-146.

29. Jyothi, N.K.; Kumar, K.V.; Murthy, P.N. FTIR, XRD and DC Conductivity Studies of Proton Conducting Gel Polymer Electrolytes based on Polyacrylonitrile (PAN). Int. J. Chem. Tech. Res. 2014, 6, 5214-5219.

30. Adel, R.; Abdallah, T.; Moustafa, Y.M.; Al-sabagh, A.M.; Talaat, H. Effect of polymer electrolyte on the performance of natural dye sensitized solar cells. Superlattice Microst. 2015, 86, 62-67.

31. Atkins, P.; Overton, T.; Rourke, J.; Weller, M.; Armstrong, F.; Hagerman, M. Inorganic Chemistry, 5th ed.; Oxford University Press: Oxford, Great Britain, 2010; pp. 431-432.

32. Wells, A.F. Structural Inorganic Chemistry, 6th ed.; Oxford University Press: New York, NY, USA, 1984.

33. Park, S.; Yoo, S.H.; Kang, H.R.; Mu Jo, S.; John, H.I.; Lee, S. Comprehensive stabilization mechanism of electron-beam irradiated polyacrylonitrile fibers to shorten the conventional thermal treatment. Sci. Rep. 2016, 6, 1-9.

34. Yue, C.; Ye, H.; Yang, X.; Wang, K.; Miao, J.; Liu, S.; Shen, Z.; Zhang, Y. Effect of Solid Bases Catalyst on Conversion of Acrylonitrile into Acrylic Acid by Hydrothermal Reaction. In E3S Web of Conferences, volume 194, Proceedings of the 2020 5th International Conference on Advances in Energy and Environment Research (ICAEER 2020), Shanghai, China, 18-20 September 2020; Weerasinghe, R., Wu, J., Weng, C.-H., Eds.; EDP Sciences: Ulis, France, 2020.

35. ChemBuddy. Available online: https://www.chembuddy.com/?left=BATE\&right=dissociation_constants\#Kb (accessed on 17 August 2021).

36. Teoh, H.; Mac, I.D.; Metz, P., Jr. Electrical conductivity of doped polyacrylonitrile (PAN). J. Phys. Colloq. 1983, 44, 687-691.

37. Kim, H.S.; Cho, H.H. Crystalline structure of polyacrylonitrile-iodine complex. J. Appl. Polym. Sci. 1994, 53, 1403-1413. [CrossRef]

38. Jyothi, N.K.; Venkataratnam, K.K.; Murty, P.N.; Kumar, K.W. Preparation and characterization of PAN-KI complexed gel polymer electrolytes for solid-state battery applications. Bull. Mater. Sci. 2016, 39, 1047-1055. [CrossRef]

39. Sahyoun, T.; Arrault, A.; Schneider, R. Amidoximes and Oximes: Synthesis, Structure, and Their Key Role as NO Donors. Molecules 2019, 24, 2470. [CrossRef] 
40. Yoshimura, A.; Zhdankin, V.V. Oxidative cyclizations of oximes using hypervalent iodine reagents. Arkivoc 2017, 1, 99-116. [CrossRef]

41. Zhang, W.; Wang, M.; Zhang, W.; Liu, W.; Yang, C.; Shen, R.; Wu, G. Significantly reduced pre-oxidation period of PAN fibers by continuous electron beam irradiation: Optimization by monitoring radical variation. Polym. Degrad. Stabil. 2018, 158, 72-82. [CrossRef]

42. Fkzer, E.; Miller, D.J. The influence of oxygen on the chemical reactions during stabilization of PAN as carbon fiber precursor. Carbon 1975, 13, 63-69.

43. Kharasch, M.S.; Sosnovsky, G. Oxidative reactions of nitriles-I autoxidation. Tetrahedron 1958, 3, 97-104. [CrossRef]

44. Basel, Y.; Hassner, A. An Improved Method for Preparation of Nitrile Oxides from Nitroalkanes for In Situ Dipolar Cyclo additions. Synthetis 1997, 3, 309-312.

45. Ajay Kumar, K.; Govindaraju, M.; Jayaroopa, P.; Vasanth Kumar, G. Nitrile oxides: A key intermediate in organic synthesis. IJPCBS 2012, 3, 91-101.

46. Belen'kii, L.I. Nitrile Oxides. Nitrile oxides, nitrones, and nitronates in organic synthesis: Novel Strategies in Synthesis, 2nd ed.; John Wiley \& Sons, Inc.: Hoboken, NJ, USA, 2008; pp. 1-127.

47. Grundmann, C.; Grünanger, P. The Nitrile Oxides: Versatile Tools of Theoretical and Preparative Chemistry. In Organische Chemie in Einzeldarstellungen, 1st ed.; Bredereck, H., Hafner, K., Miiller, E., Eds.; Publisher Springer-Verlag Berlin: New York, NY, USA, 1971; Volume 13, pp. 10-20.

48. Zhang, W.; Lin, J.-H.; Zhang, P.; Xiao, J.-C. A convenient reagent for the conversion of aldoximes into nitriles and isonitriles. Chem. Commun. (Camb) 2020, 56, 6221-6224. [CrossRef]

49. Popescu, V.; Astanei, D.-G.; Burlica, R.; Popescu, A.; Munteanu, C.; Ciolacu, F.; Ursache, M.; Ciobanu, L.; Cocean, A. Sustainable and cleaner microwave-assisted dyeing process for obtaining eco-friendly and fluorescent acrylic knitted fabrics. J. Clean. Prod. 2019, 232, 451-461.

50. Alarifi, I.M.; Alharbi, A.; Khan, W.S.; Swindle, A.; Asmatulu, R. Properties of electrospun polyacrylonitrile nanofibers for structural health monitoring. Materials 2015, 8, 7017-7031. [CrossRef] [PubMed]

51. Zhang, L.; Dai, Y.; Kai, Y.; Jin, R. Structural evolution and kinetic study of high isotacticity poly(acrylonitrile) during isothermal pre-oxidation. Carbon Lett. 2011, 12, 229-235. [CrossRef]

52. Setnescu, R.; Jipa, S.; Setnescu, T.; Kappel, W.; Kobayashi, S.; Osawa, Z. IR and X-ray characterization of the ferromagnetic phase of pyrolyzed polyacrylonitrile. Carbon 1999, 37, 1-6. [CrossRef] 